Supplement of Biogeosciences, 15, 4405-4429, 2018

https://doi.org/10.5194/bg-15-4405-2018-supplement

(C) Author(s) 2018. This work is distributed under

the Creative Commons Attribution 4.0 License.

(c) (1)

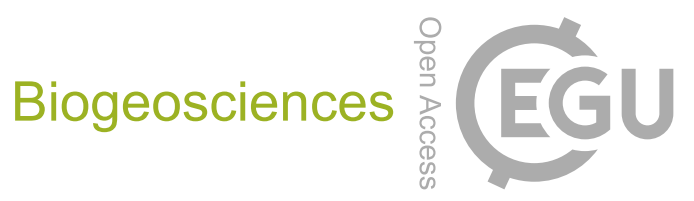

Supplement of

\title{
Modeling seasonal and vertical habitats of planktonic foraminifera on a global scale
}

\section{Kerstin Kretschmer et al.}

Correspondence to: Kerstin Kretschmer (kkretschmer@marum.de)

The copyright of individual parts of the supplement might differ from the CC BY 4.0 License. 


\section{Contents of this file}

Figure S1 (page 2): 50-year (left panel) and 300-year (right panel) time series of the year-to-year difference (in $\mathrm{mmolm}^{-3}$ ) of the modeled carbonate ion concentration $\left(\mathrm{CO}_{3}^{2-}\right.$; grey), dissolved inorganic nitrate $\left(\mathrm{NO}_{3}^{-}\right.$; light blue), small phytoplankton concentration (orange), zooplankton concentration (magenta), and the concentration of $N$. pachyderma (black) at the surface ocean, $105 \mathrm{~m}, 250 \mathrm{~m}$, and $530 \mathrm{~m}$ water depth. Note that the left panel only shows a zoom for the latter three mentioned concentrations.

Figure S2 (page 3): Modeled peak timing (top row) and/or modeled peak amplitude (bottom row) vs. annual mean temperature (in ${ }^{\circ} \mathrm{C}$ ) averaged over the top $55 \mathrm{~m}$ of the water column for (a) N. pachyderma, (b) N. incompta, (c) G. bulloides, (d) G. ruber (white), and (e) T. sacculifer. The color coding corresponds to latitude. Modeled peak timing is given in months and modeled peak amplitudes have been log-transformed. Note that peak timings of each species from the southern hemisphere have been transformed to northern hemisphere equivalents by adding or subtracting 6 months. For a better visualization the peak timing data has been offset along the ordinate axis to avoid that overlapping points plot on top of each other (this has been achieved by adding a small amount of white noise to the peak timing data). The grey shadings in the top row panels show the data density, i.e., where most of the data points occur.

Figure S3 (pages 4-14): Comparison of export planktonic foraminiferal shell fluxes in sediment traps (in $\log _{10}\left[\# \mathrm{~m}^{-2}\right.$ day $\left.{ }^{-1}\right]$; grey triangles) with the residuals (i.e., the deviation from the mean) of the volume integrated modeled biomass (in $\mathrm{mmol} \mathrm{Cm}^{-3} \times 10^{-4} ;$ light blue squares). The respective location of each sediment trap is given in Table S1.

Figure S4 (pages 15-26): Comparison of the vertical distribution of live specimens in plankton tows (in \# $\mathrm{m}^{-3}$; grey bars) with modeled concentrations over depth (in mmol $\mathrm{Cm}^{-3}$; light blue profiles). Dashed dark grey and blue lines indicate average living depth (in $\mathrm{m}$ ) and vertical dispersion calculated for the plankton tows $\left(\mathrm{ALD}_{\text {tow }} \pm \mathrm{VD}_{\text {tow }}\right)$ and PLAFOM2.0 ( $\left.\mathrm{ALD}_{\bmod } \pm \mathrm{VD}_{\text {mod }}\right)$, respectively. The respective location of each plankton tow sample is given in Table S2.

Figure S5 (page 27): Difference in potential temperature (in ${ }^{\circ} \mathrm{C}$ ) between the Community Earth System Model, version 1.2.2 (CESM1.2) and the World Ocean Atlas 2013 (WOA13; Locarnini et al., 2013) averaged over the top (a) $55 \mathrm{~m}$ and (b) $250 \mathrm{~m}$ of the water column.

Table S1 (page 28): Information on sediment trap data.

Table S2 (pages 29-30): Information on plankton tow data.

Table S3 (pages 31-32): (a) Peak season (i.e., season of maximum production) and (b) peak amplitude (i.e., maximum in production divided by the annual mean) for each planktonic foraminiferal species at the locations of the sediment traps shown in Figure $1 \mathrm{~b}$ in the main text. Empty cells indicate absence of species in either the sediment trap data or the model output.

Table S4 (page 33): Average living depths for each planktonic foraminiferal species at the locations of the plankton tows shown in Figure 1b in the main text. Empty cells indicate if species has been absent in either the plankton tow data or the model output. 
Figure S1.
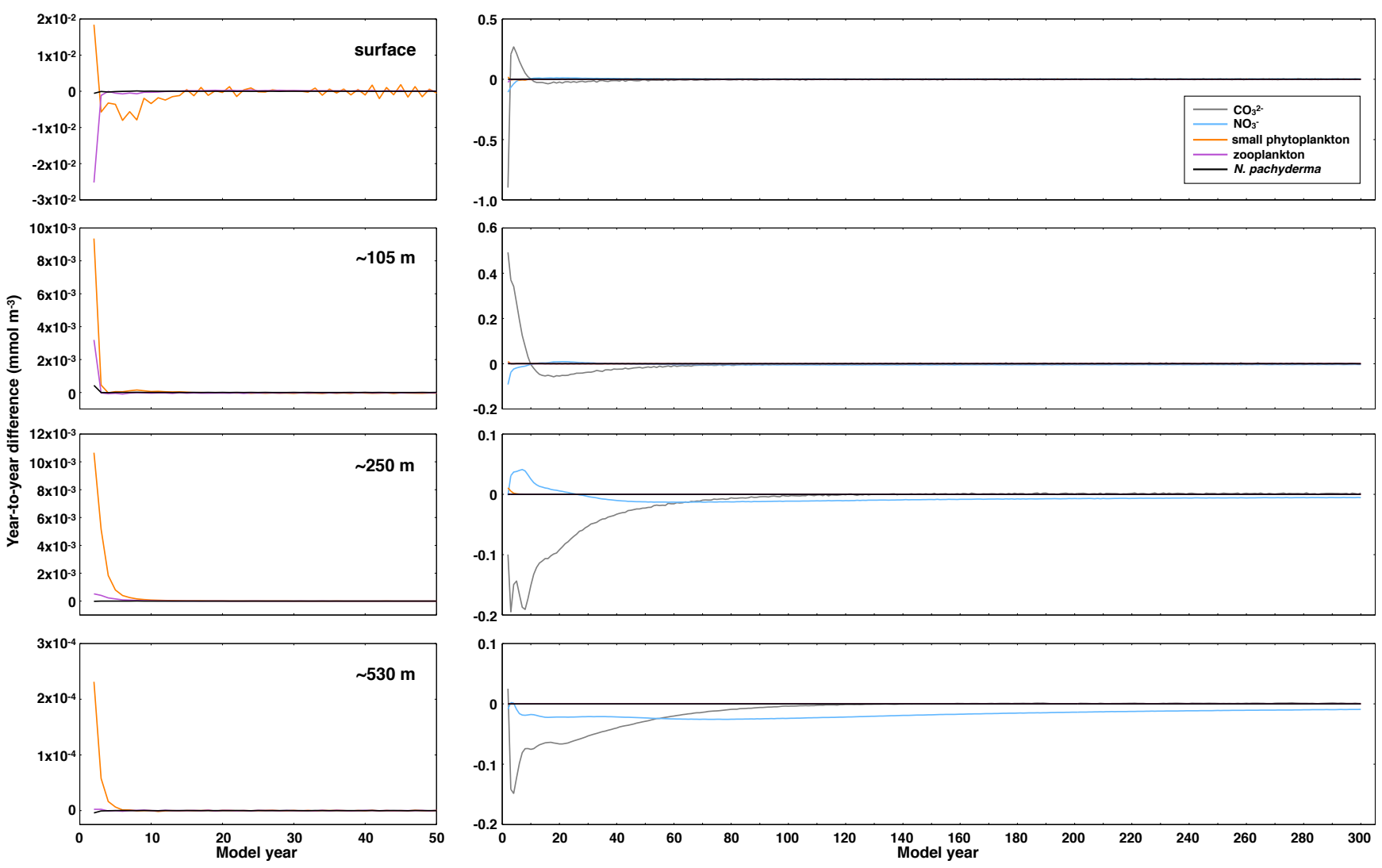
Figure S2.
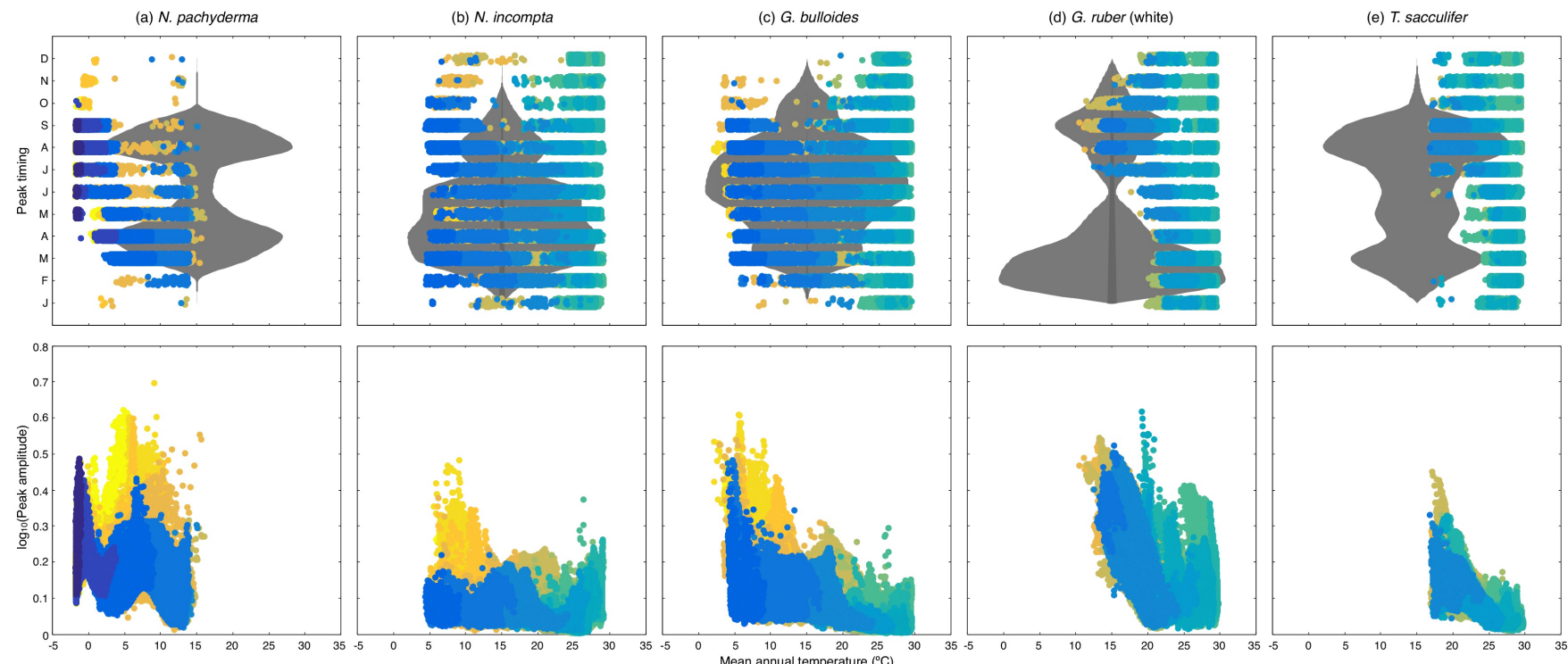

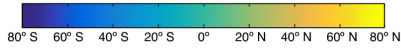


Figure S3.

Site GS2

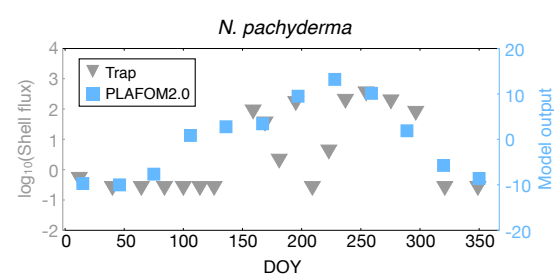

Site OG5

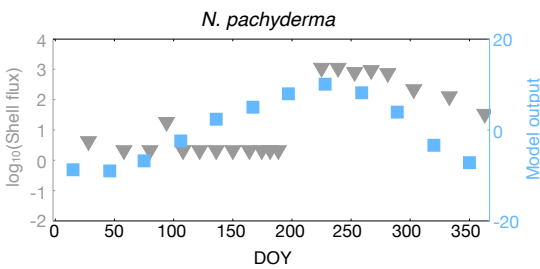

Site NB6/7
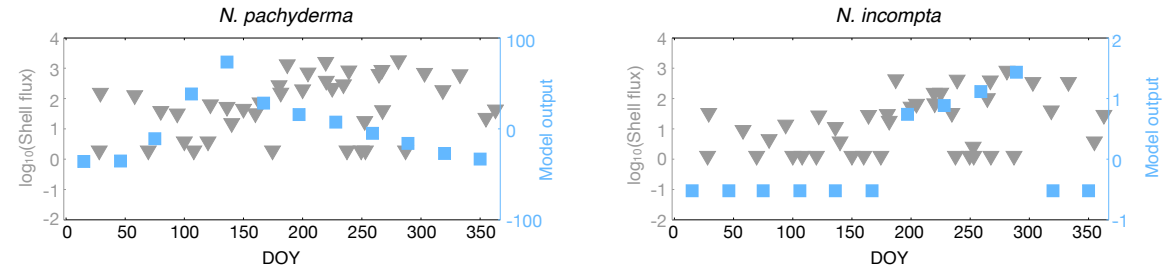

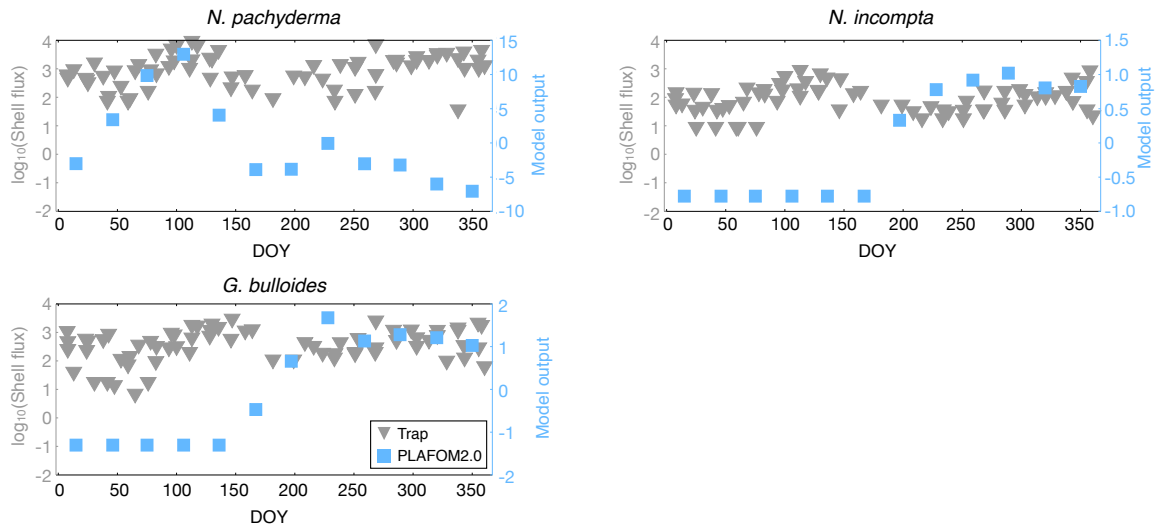

\section{Site PAPA}
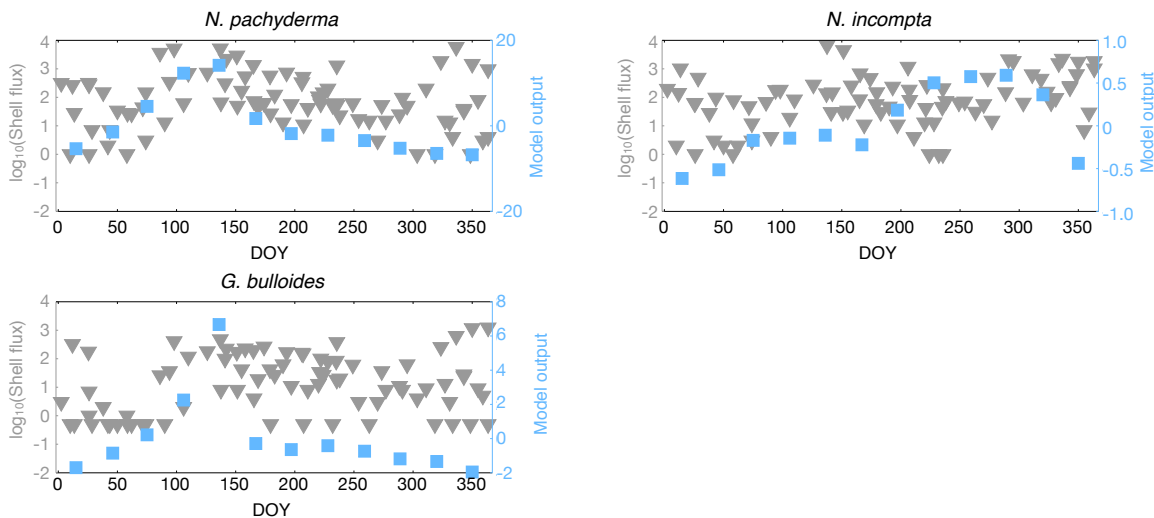

Site SA
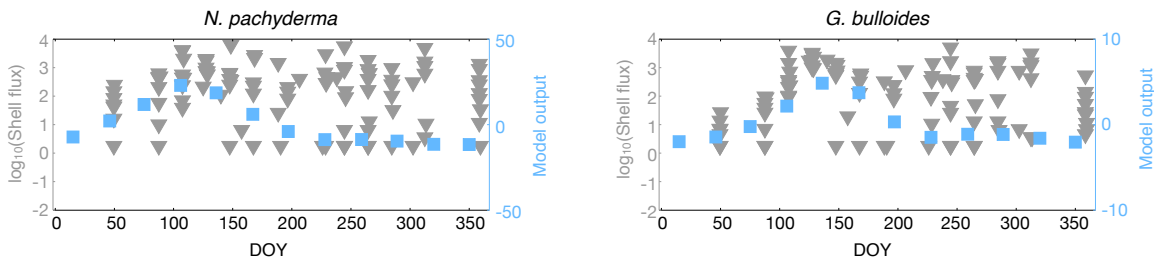


\section{Site KNOT}
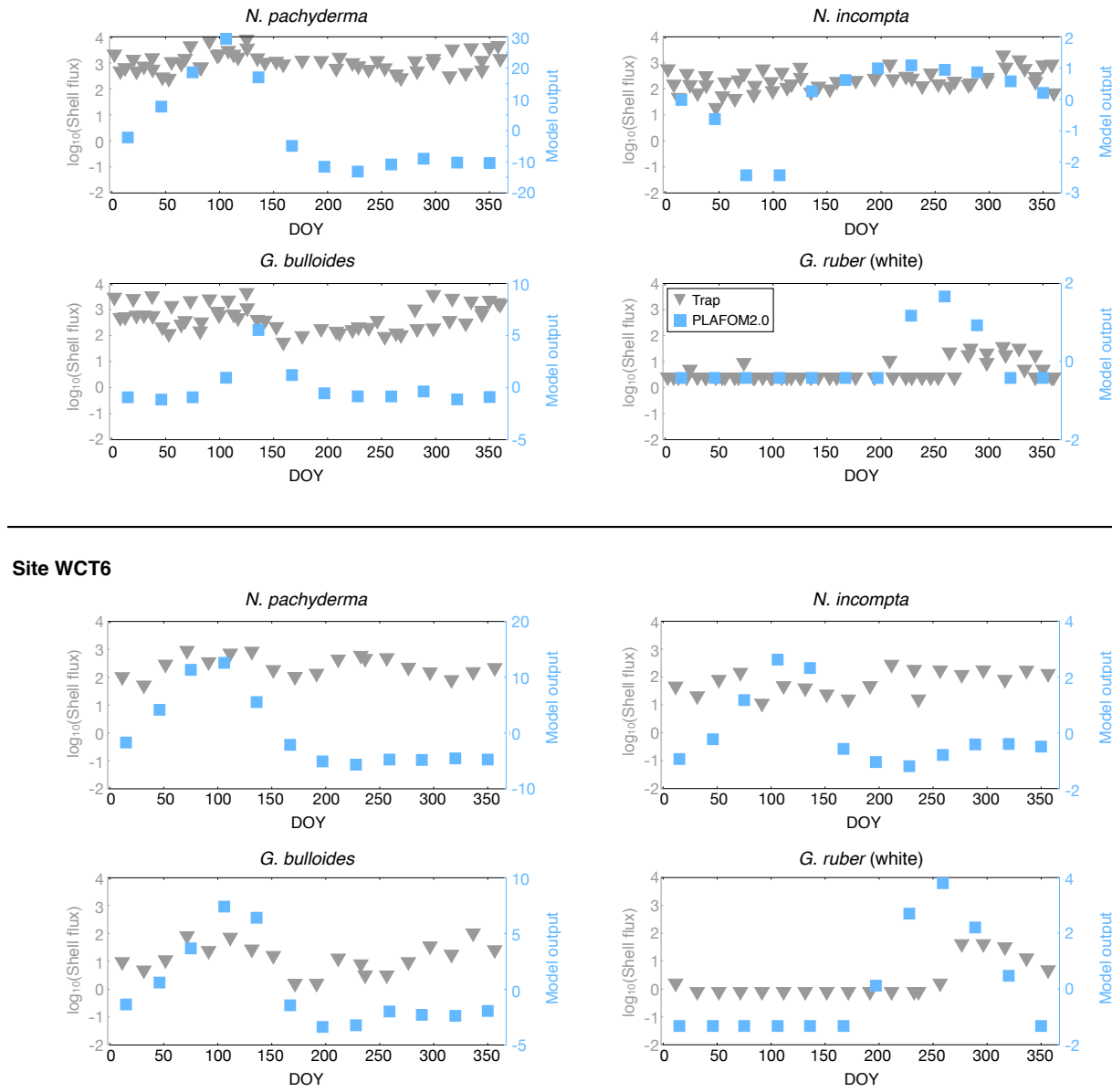
Site WCT2
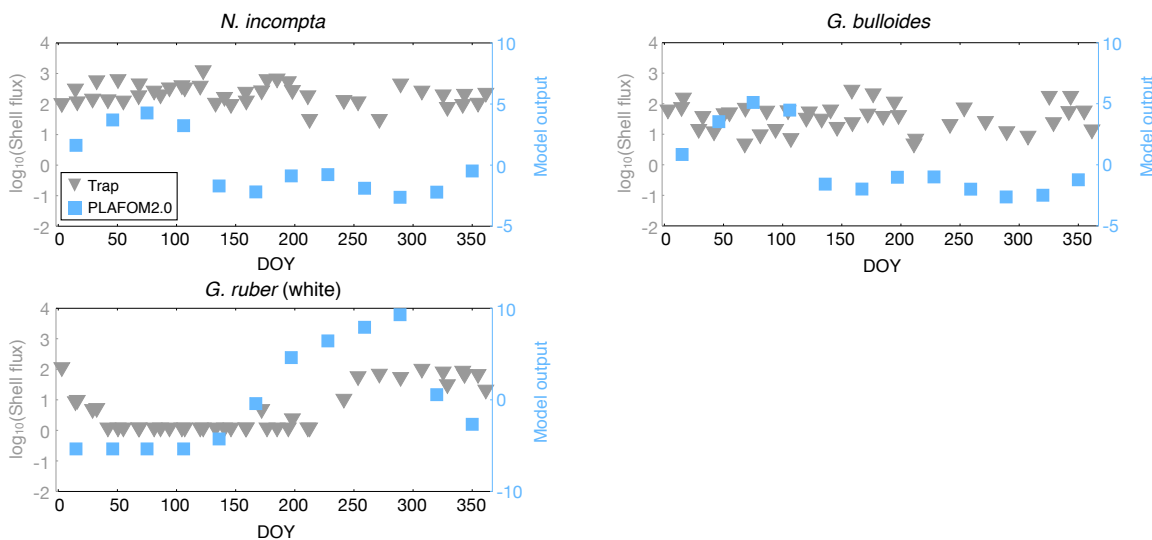

\section{Site WCT7}
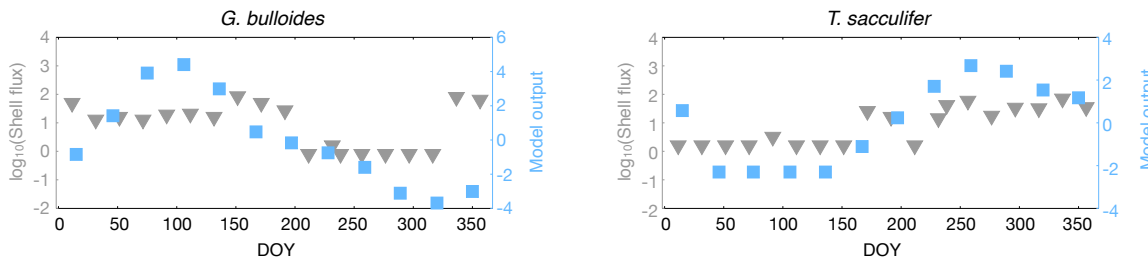

\section{Site WCT1}
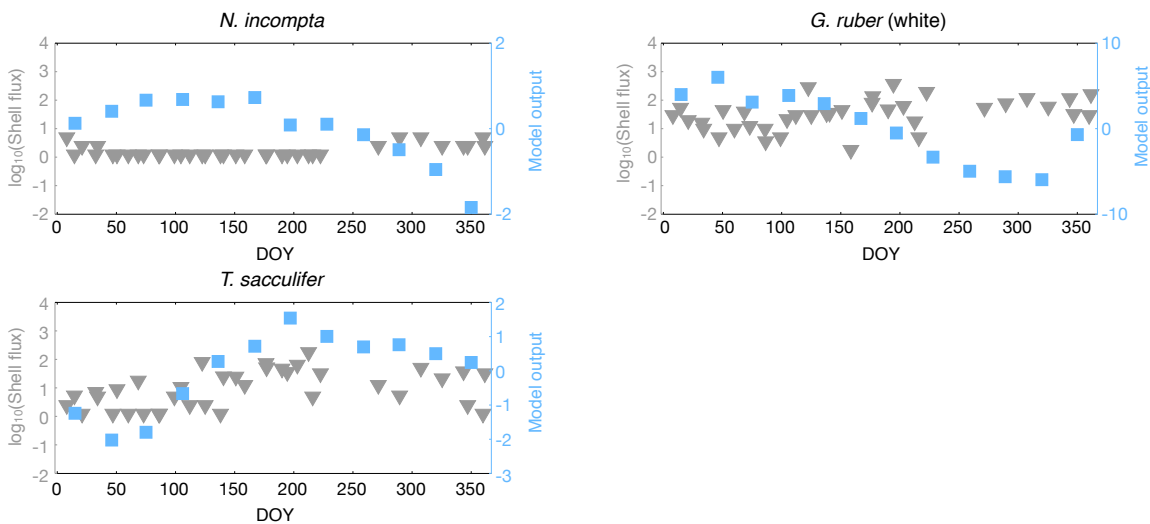

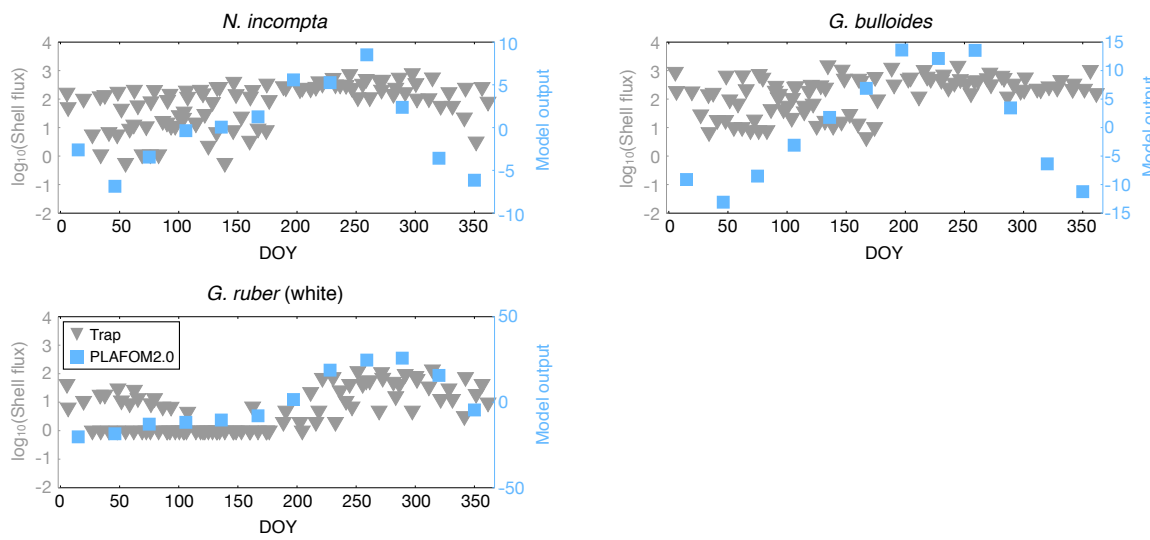

\section{Site SPB}
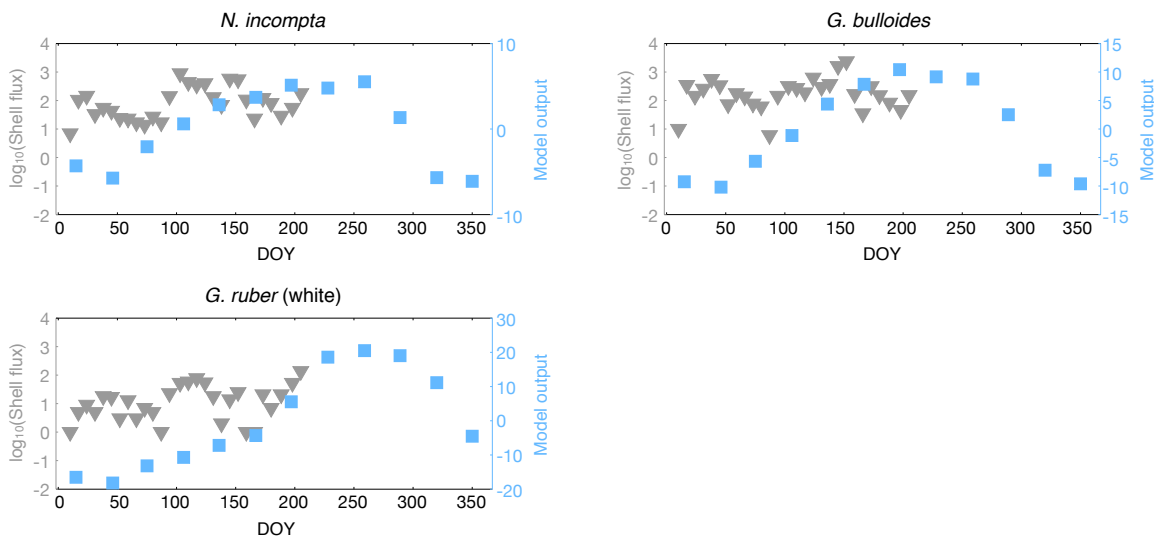

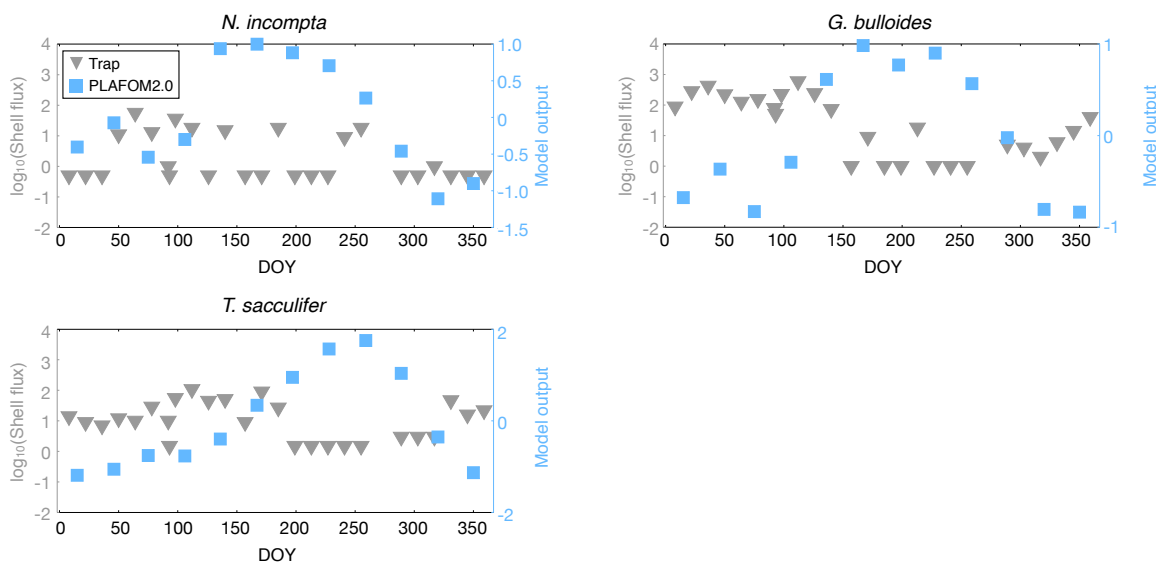

Site L1
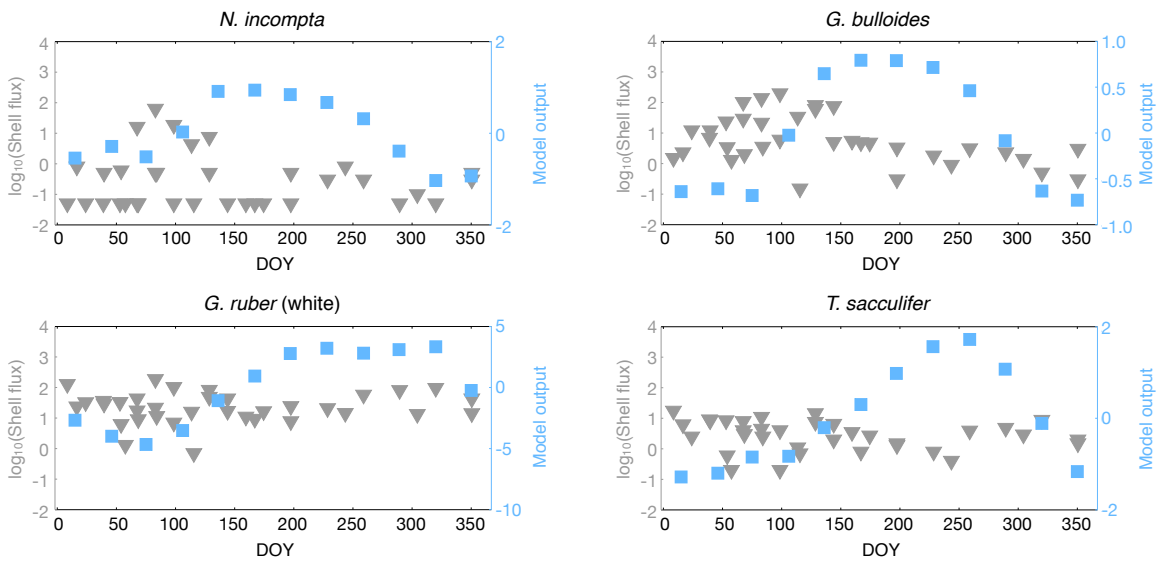

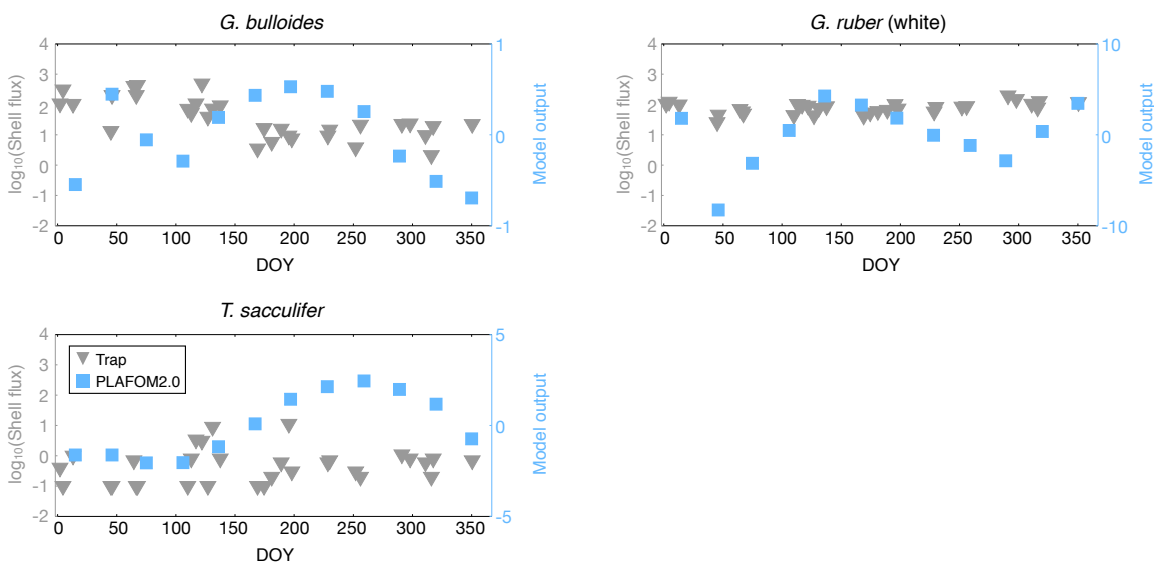

\section{Site WAST}
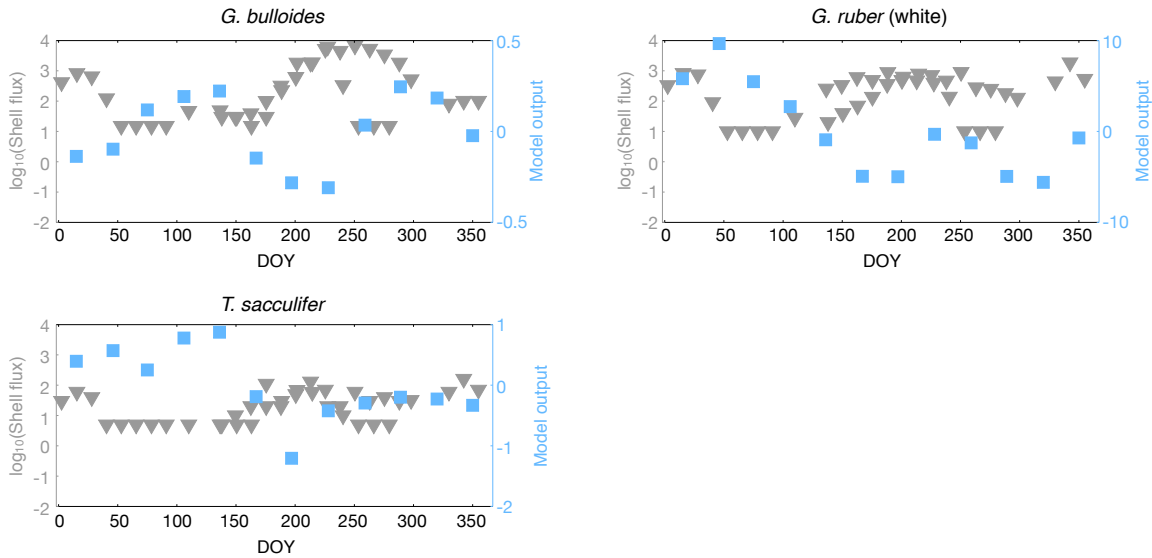


\section{Site EA1}
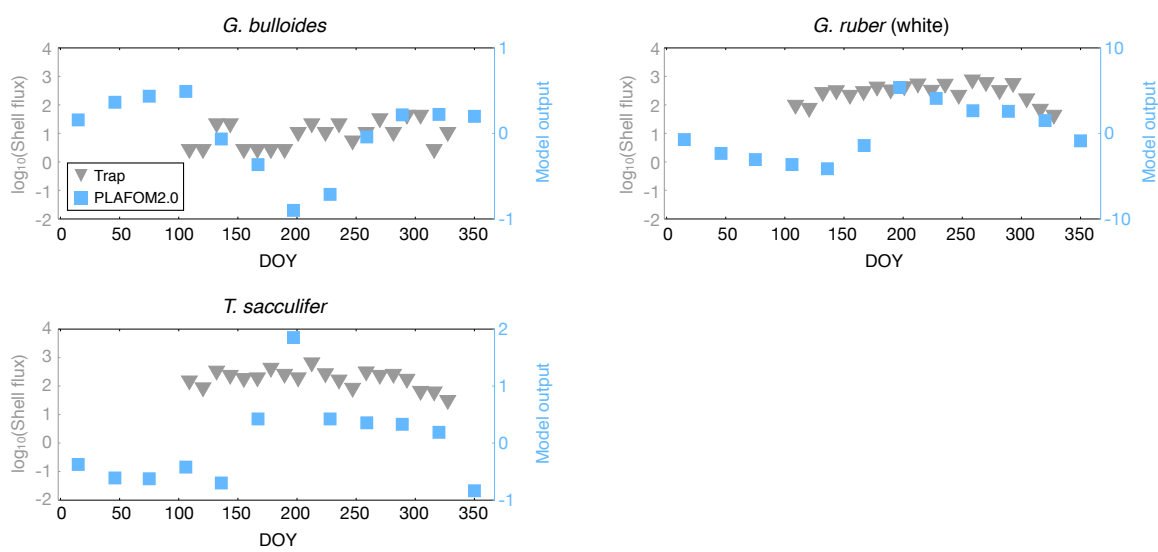

\section{Site EA2}
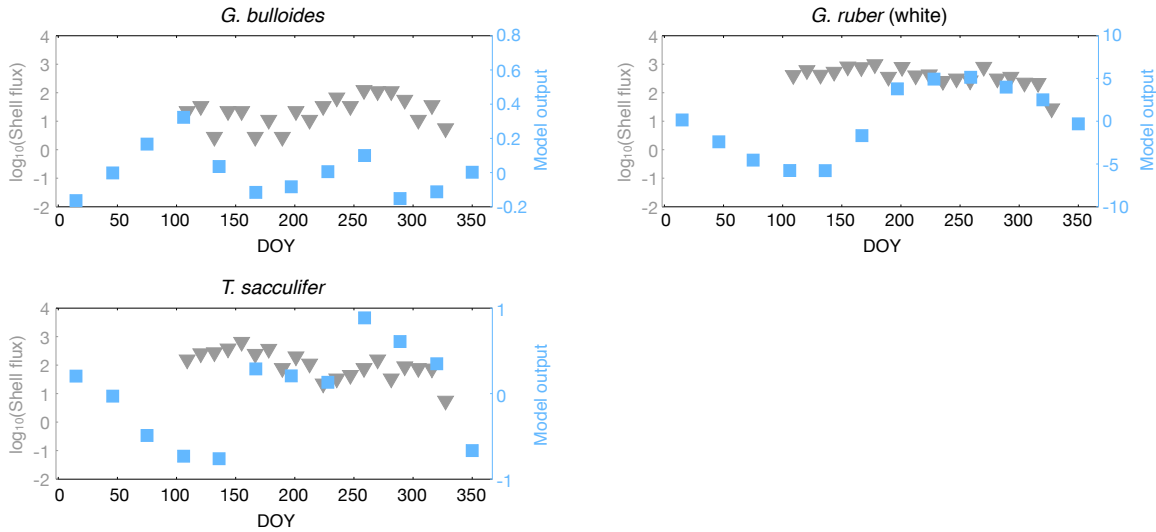

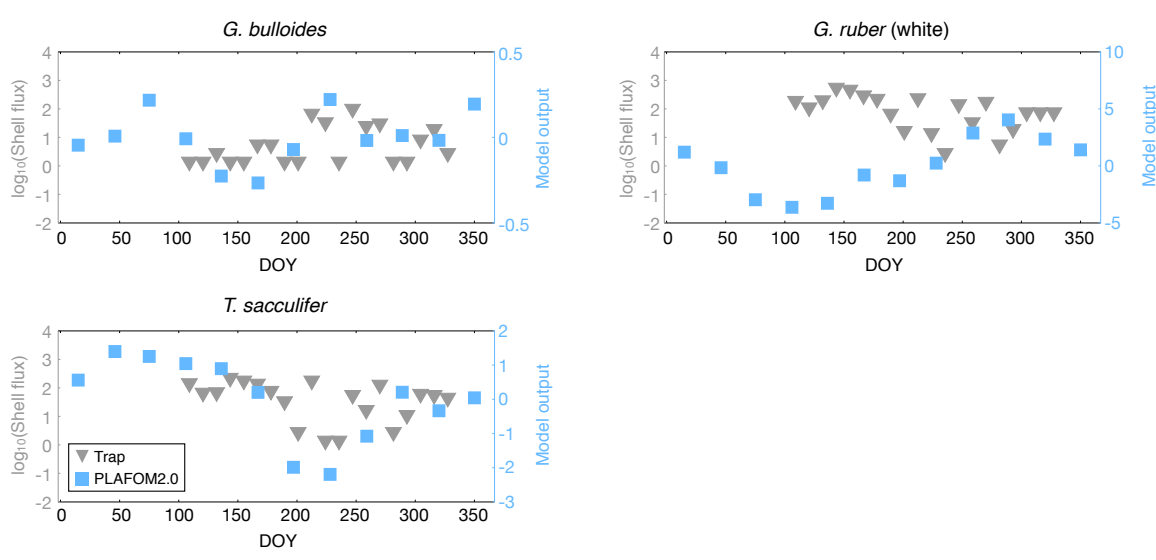

\section{Site EA4}
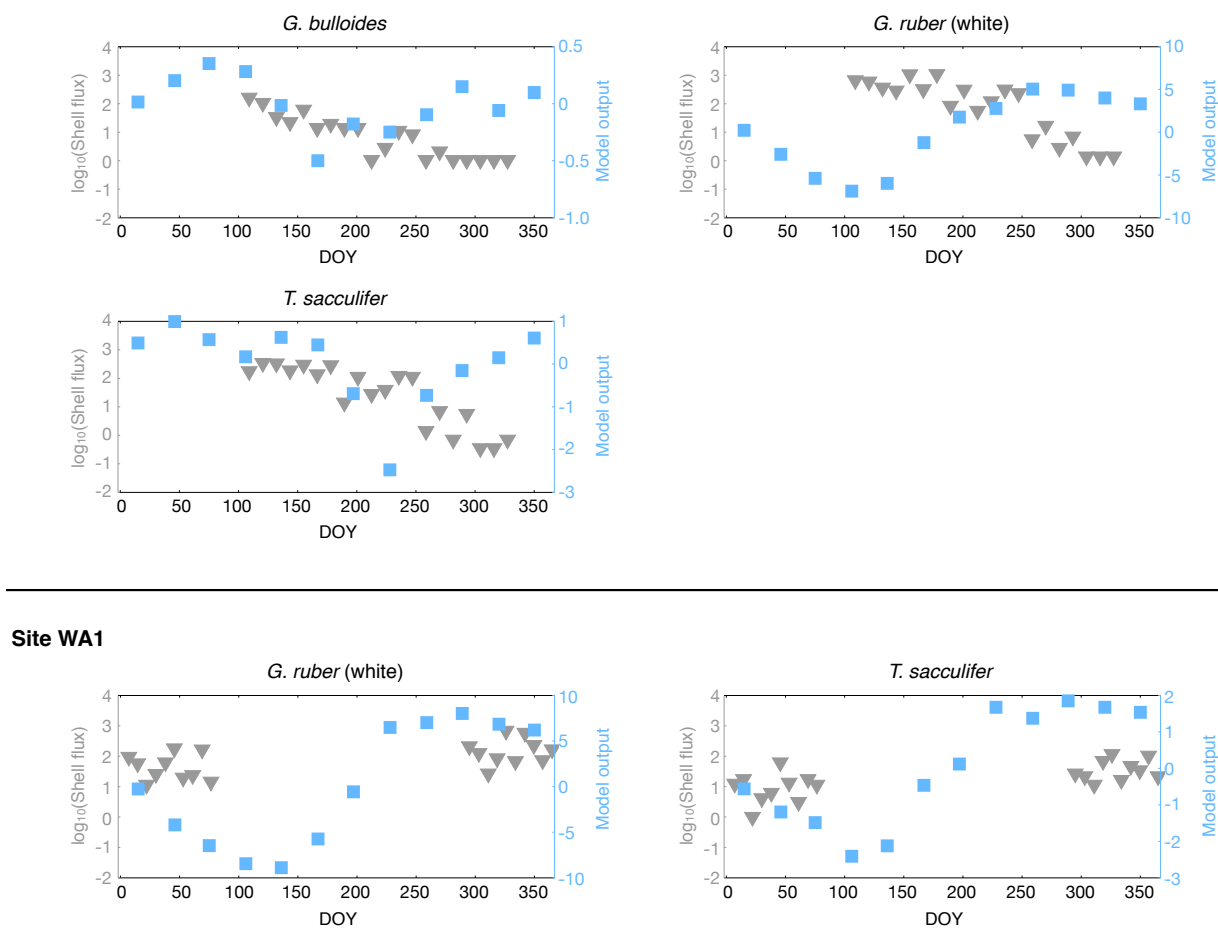
Site NCR
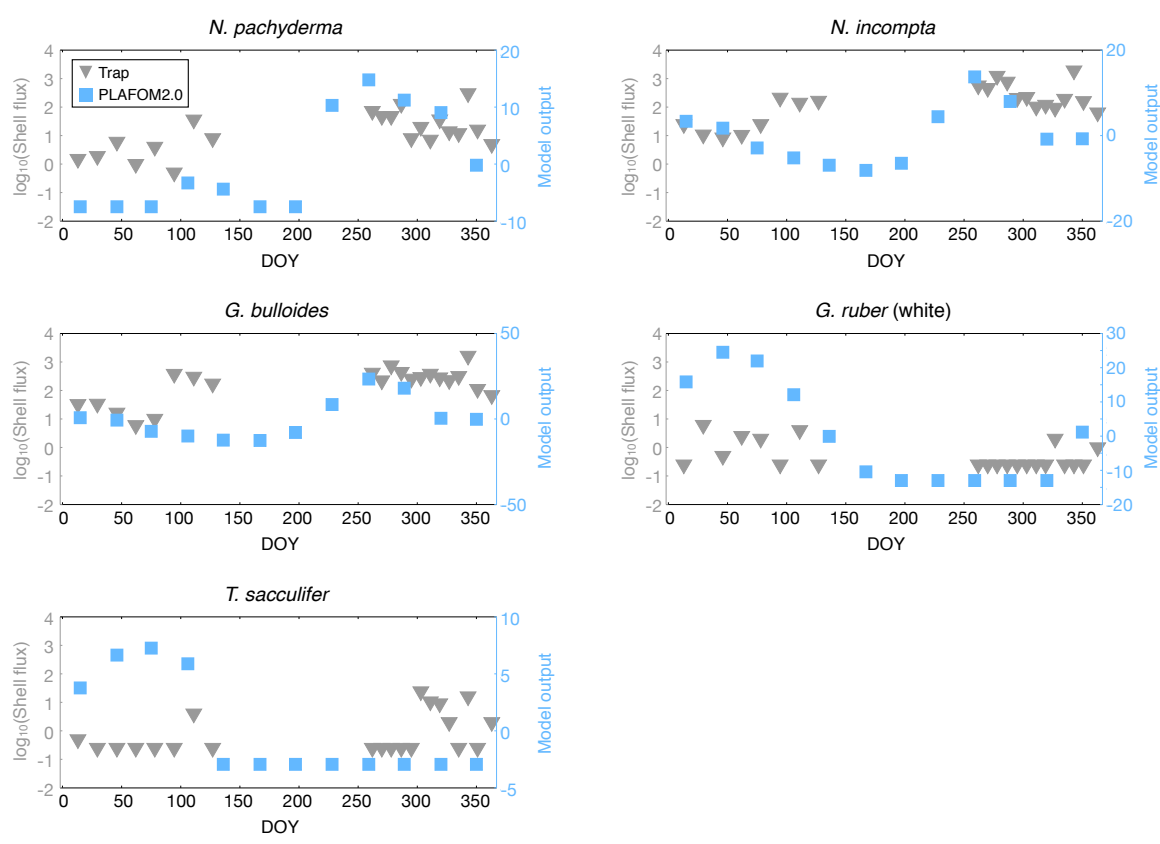

\section{Site SCR}
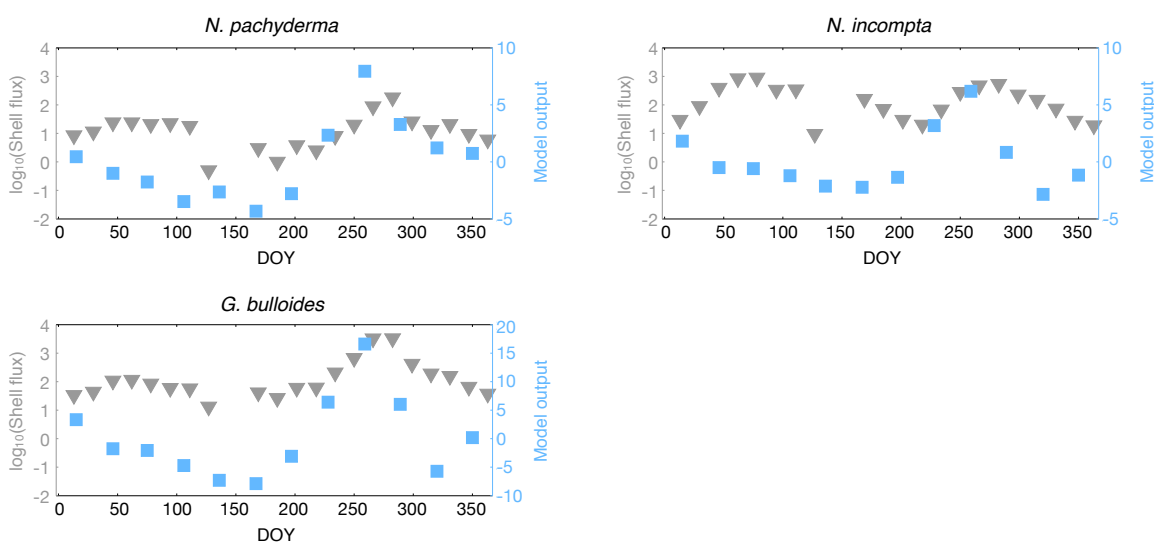
Site CP
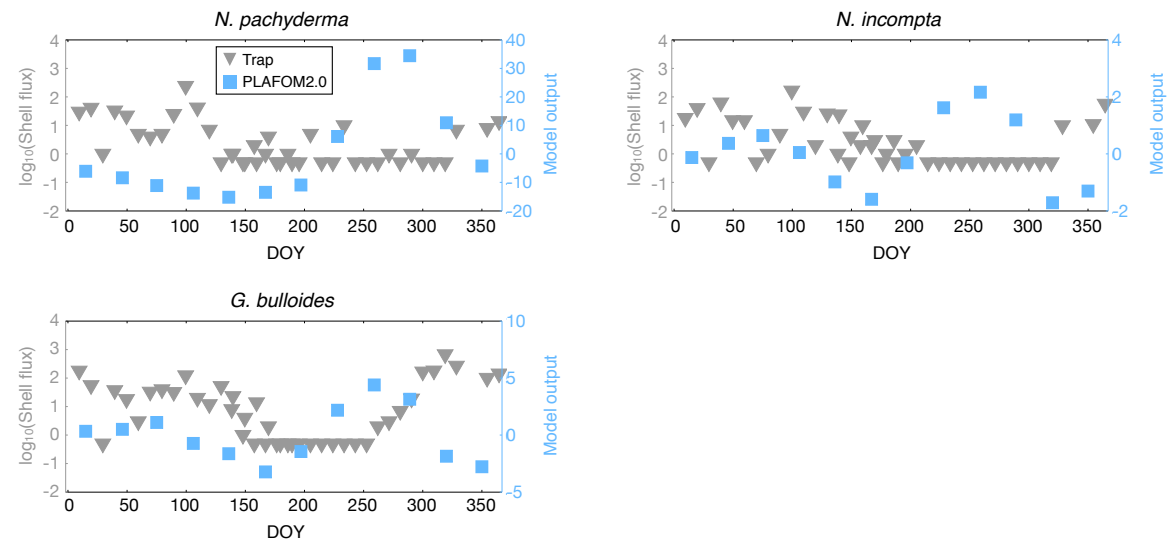

Site WS34

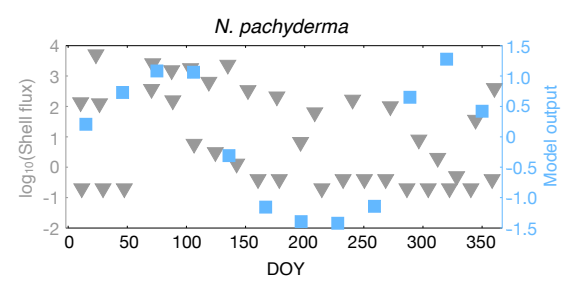


Figure S4.

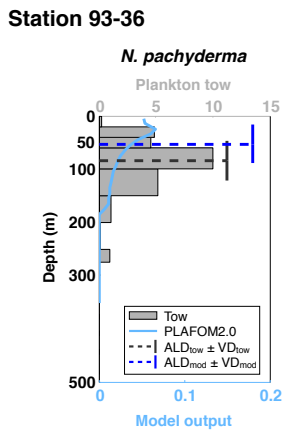

Station PS78-25

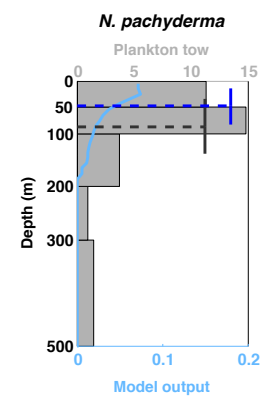

Station PS78-44

N. pachyderma

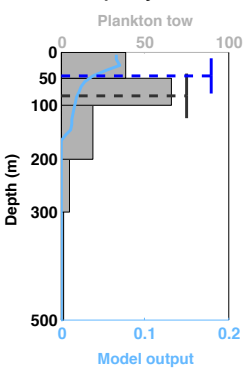

Station PS78-75

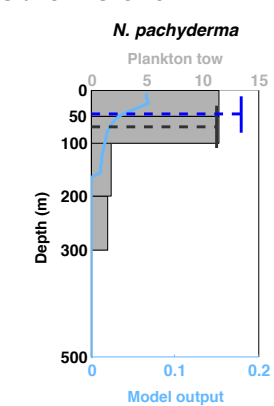


N. pachyderma

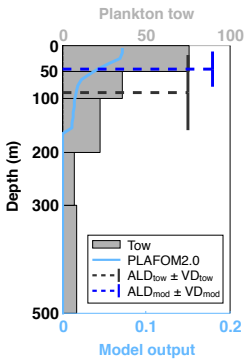

Station PS55-043

\section{N. pachyderma}

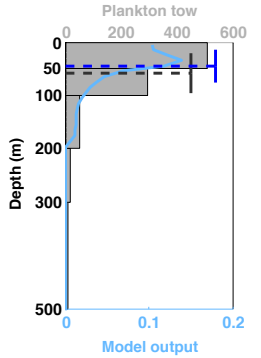

Station PS55-063

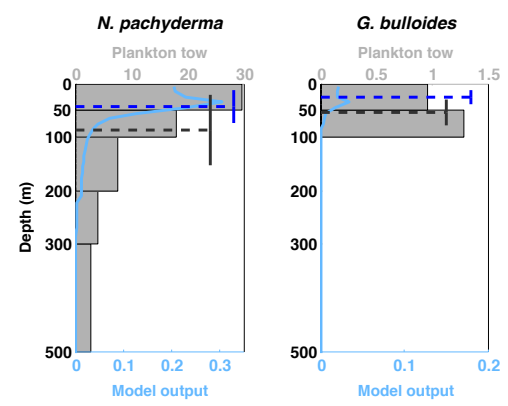




\section{Station MN116}

N. pachyderma

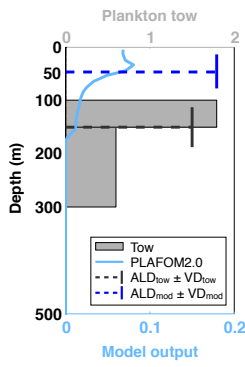

\section{Station MN2}

N. pachyderma

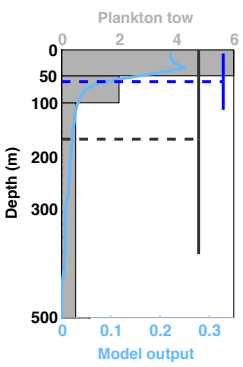

Station MN323

N. pachyderma

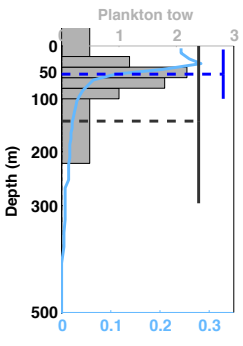

Model output

Station MN314

N. pachyderma

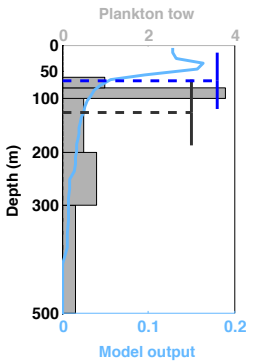



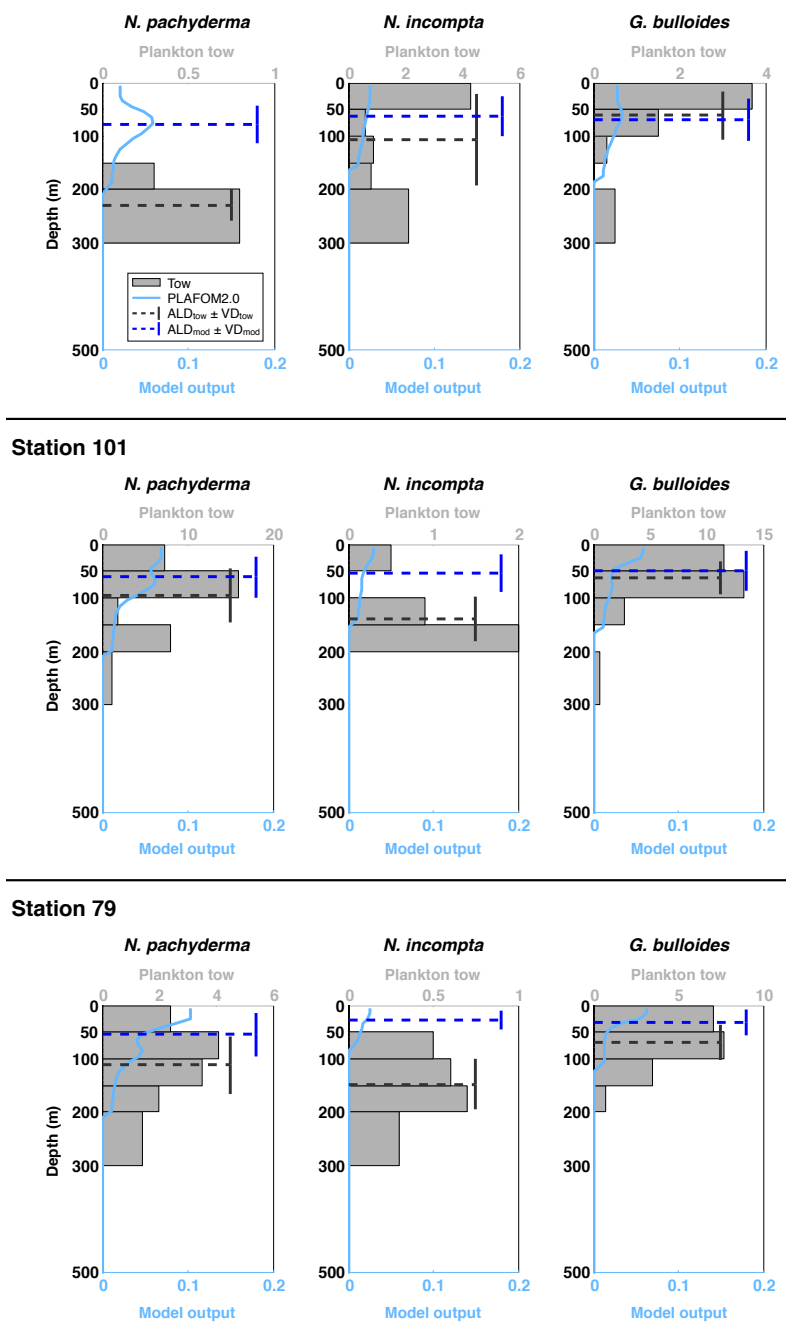

\section{Station KNOT}
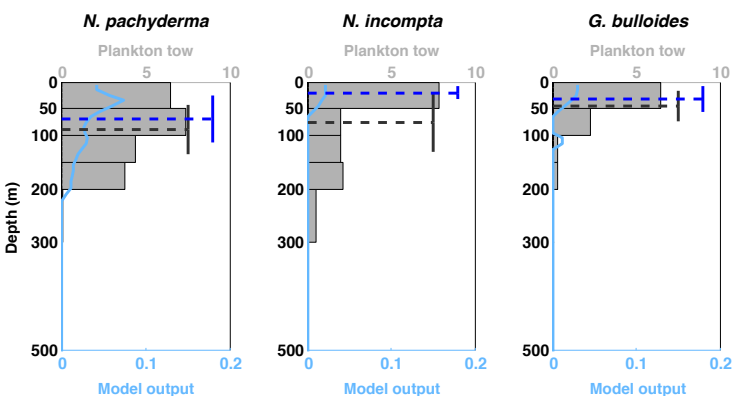


\section{Station \#B}
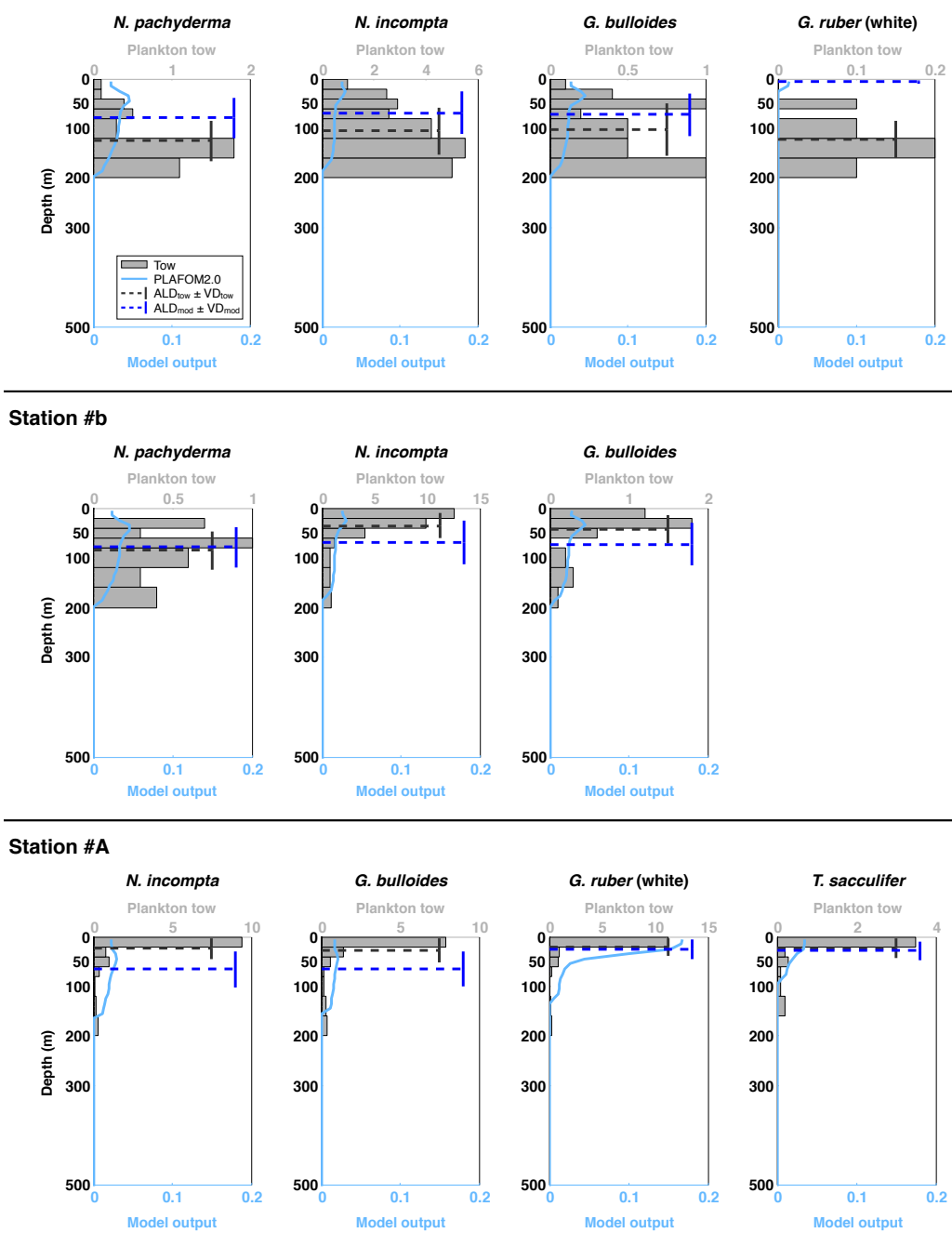

Station \#E
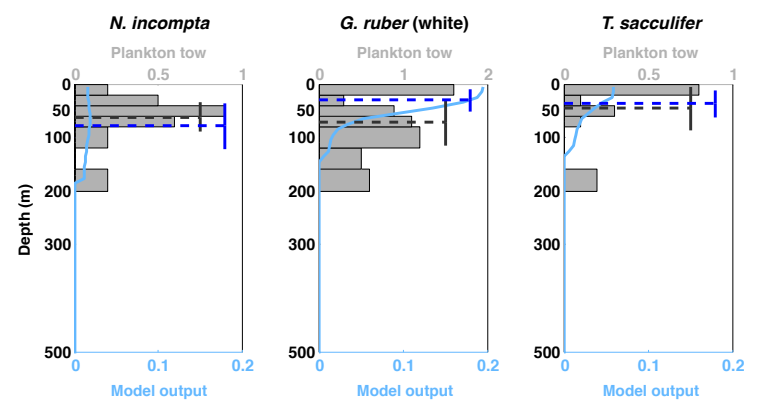

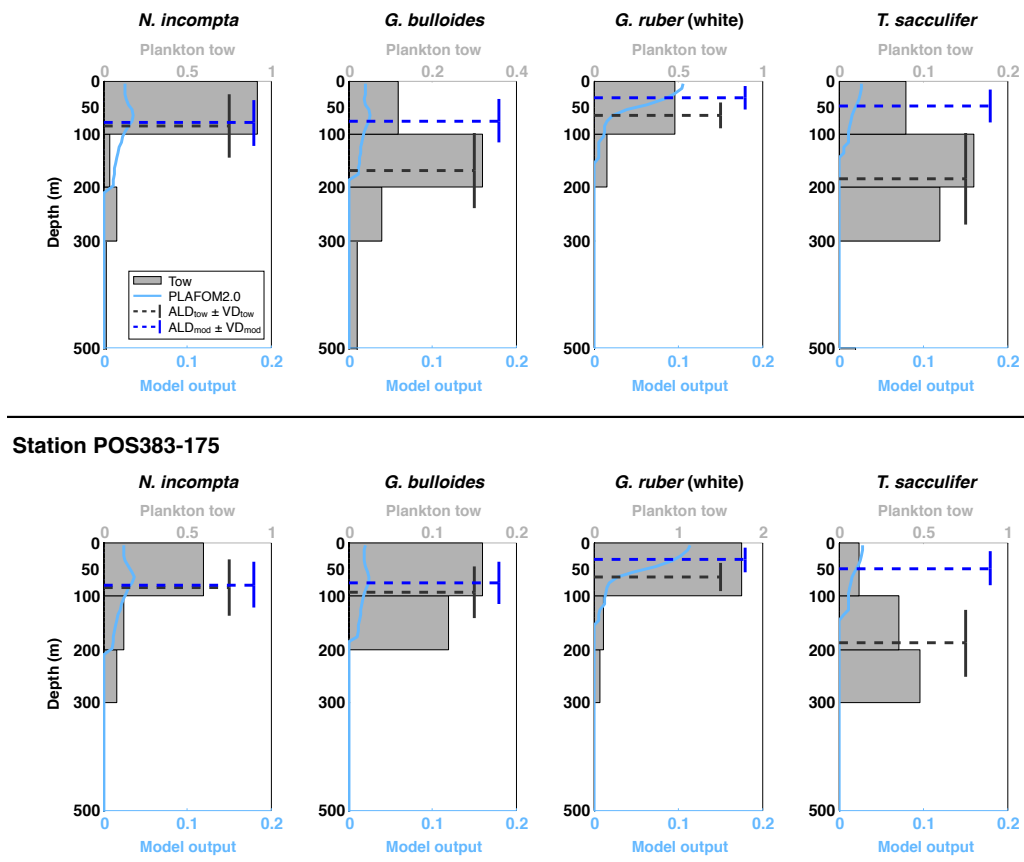

Station POS247-1389
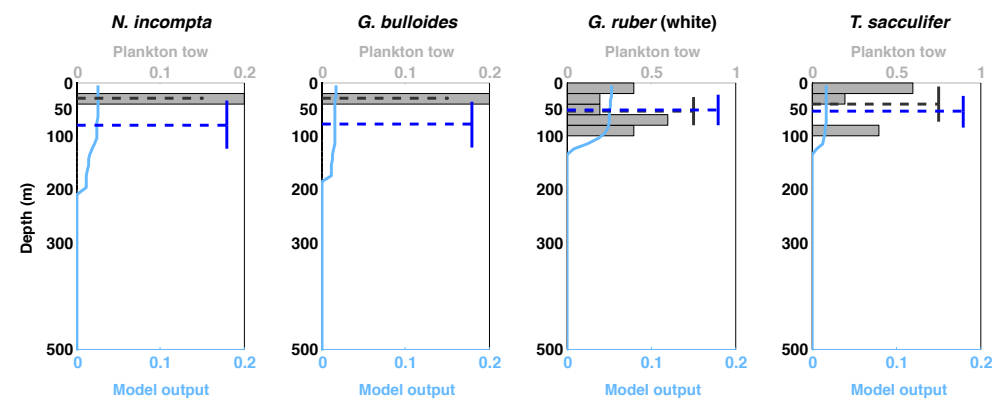
Station MOC1-38
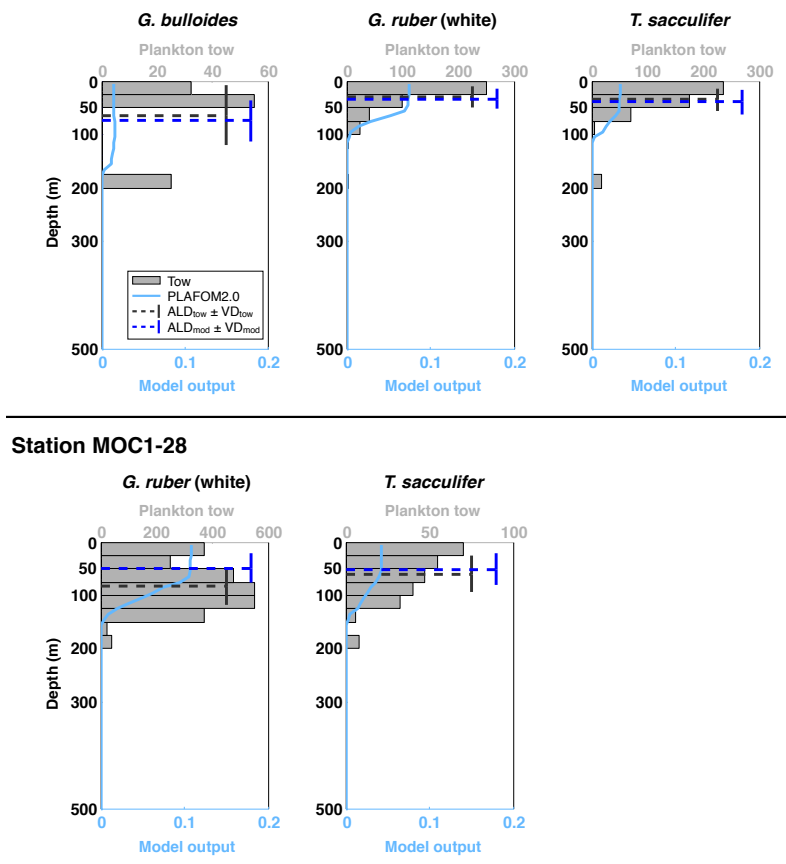

Station MOC1-23
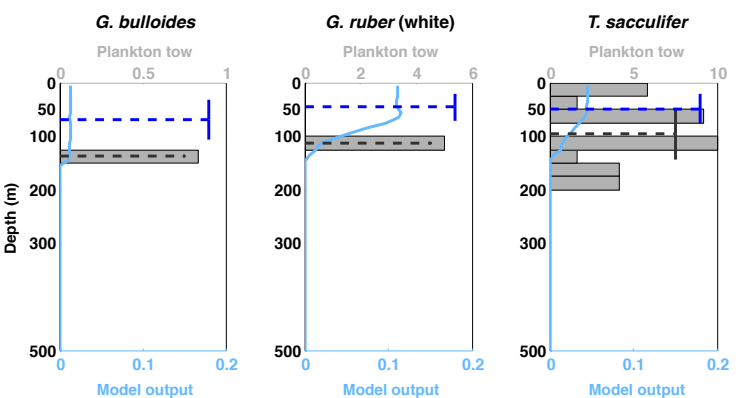

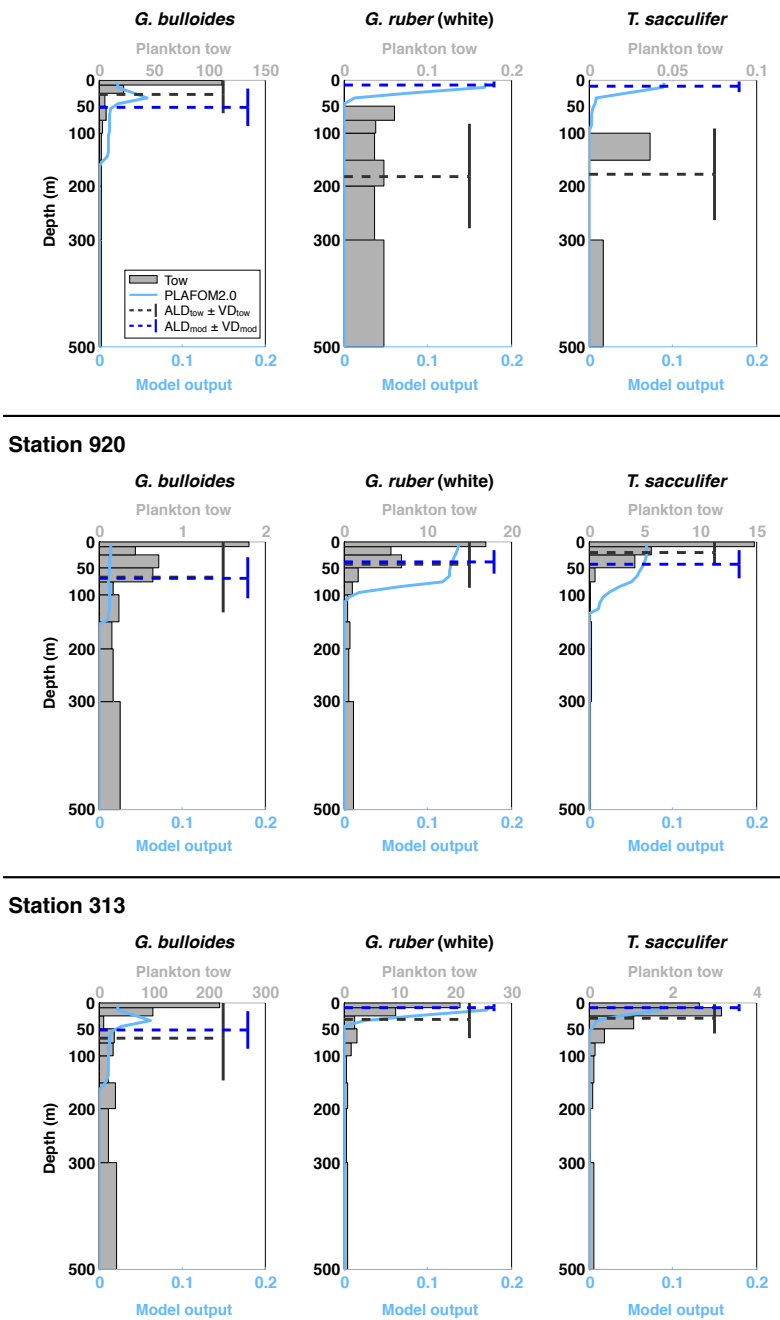

Station 917
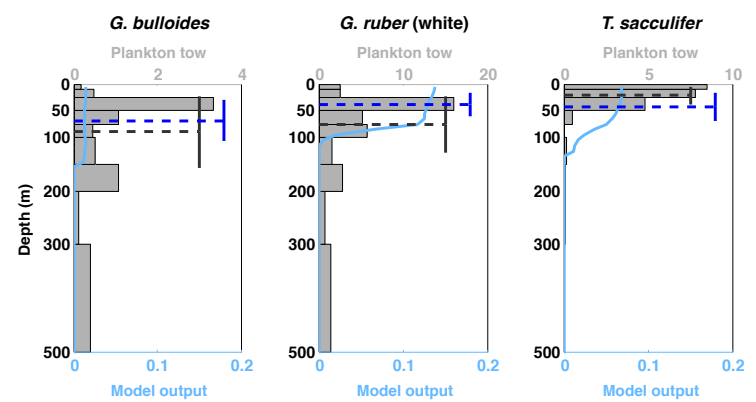
Station MOC63
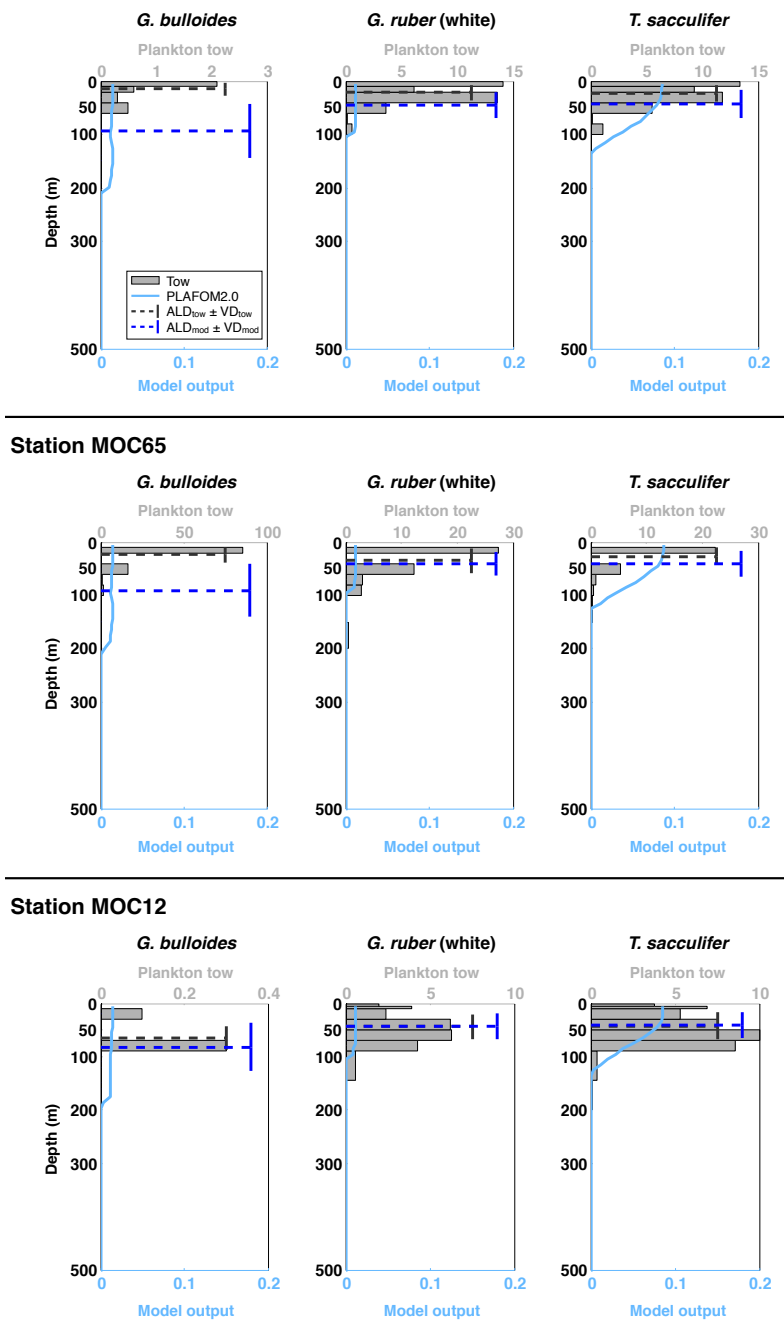

\section{Station MOC66}
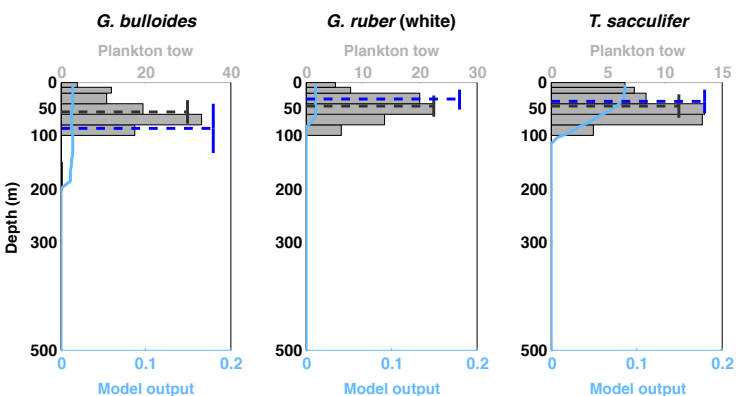
Station MOC15
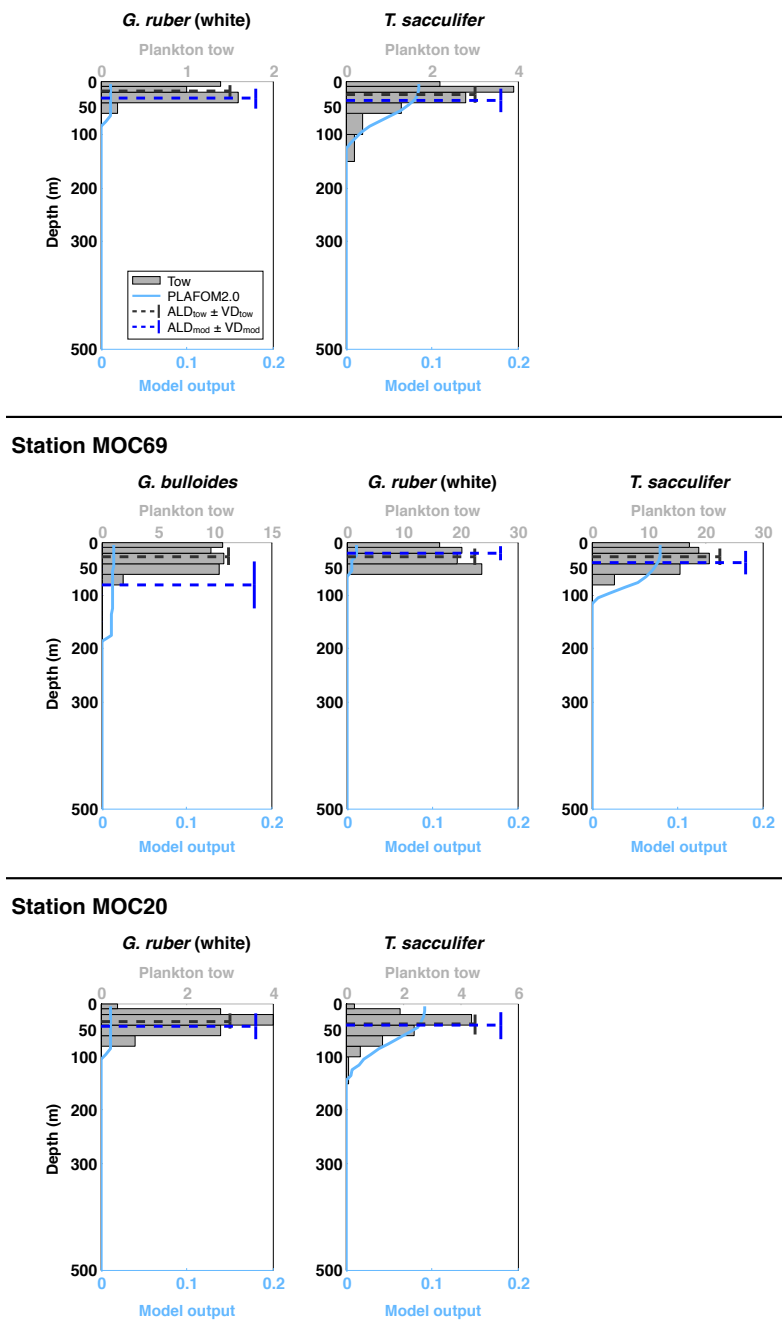

Station MOC71
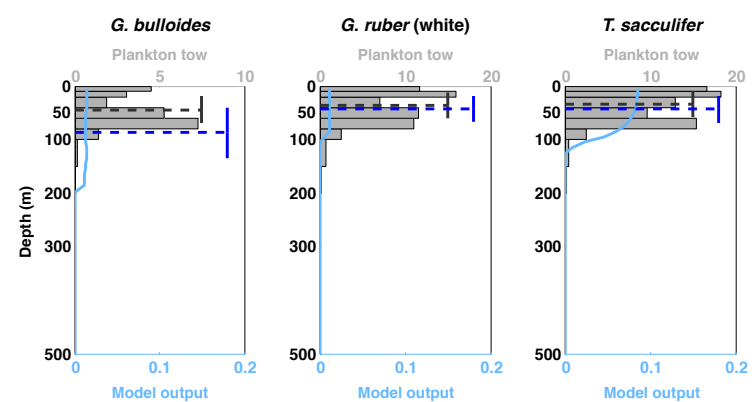
Station MOC72
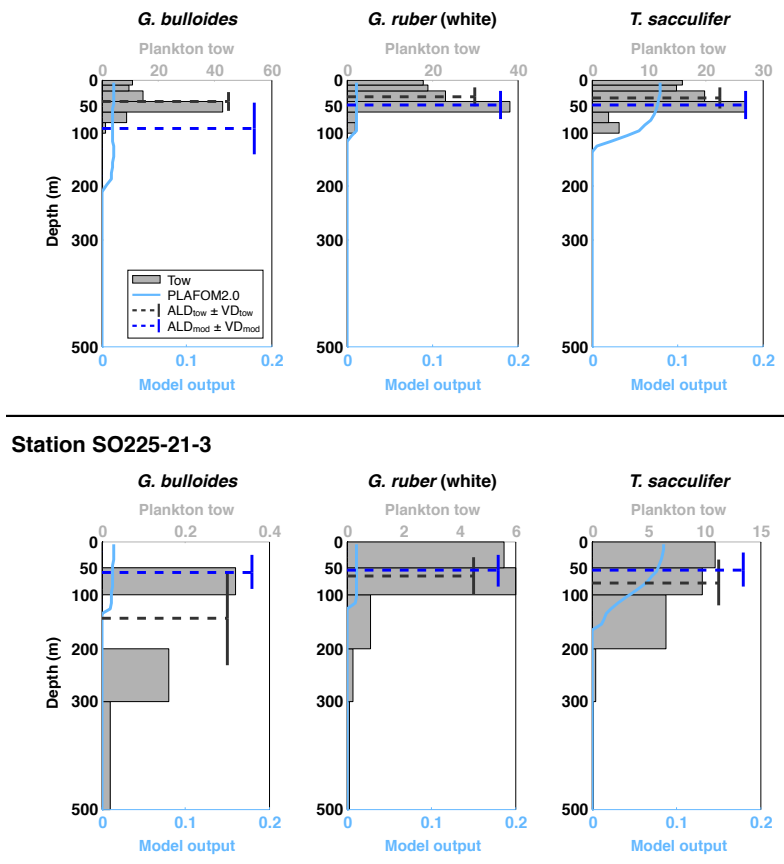

\section{Station TNO57-16}
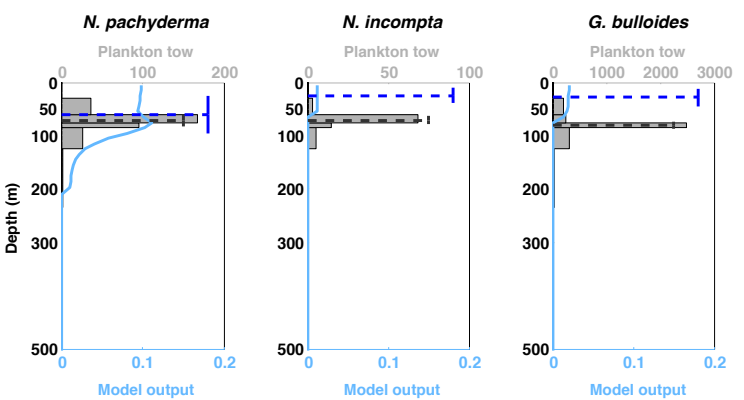

Station TNO57-13

N. pachyderma

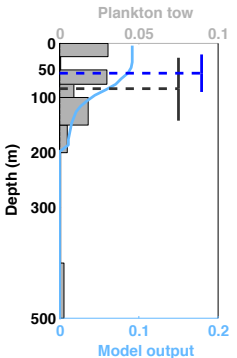


Station AN98-O

N. pachyderma

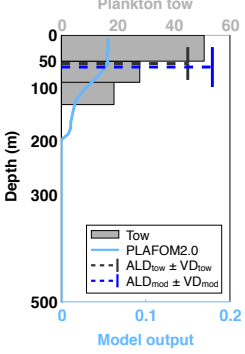

Station AN99-O

N. pachyderma

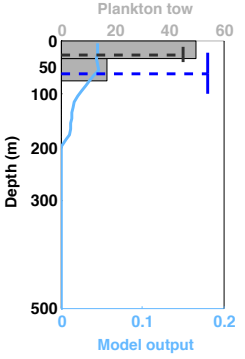

Station ANOO-O

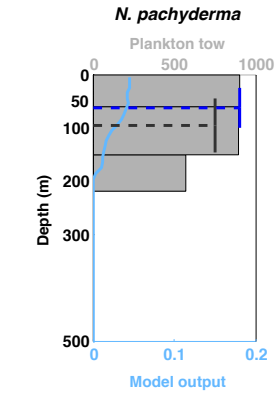

Station AN01-O

N. pachyderma

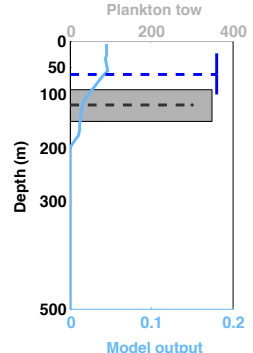


Figure S5.

(a) CESM1.2 - WOA13 (0-55 m)
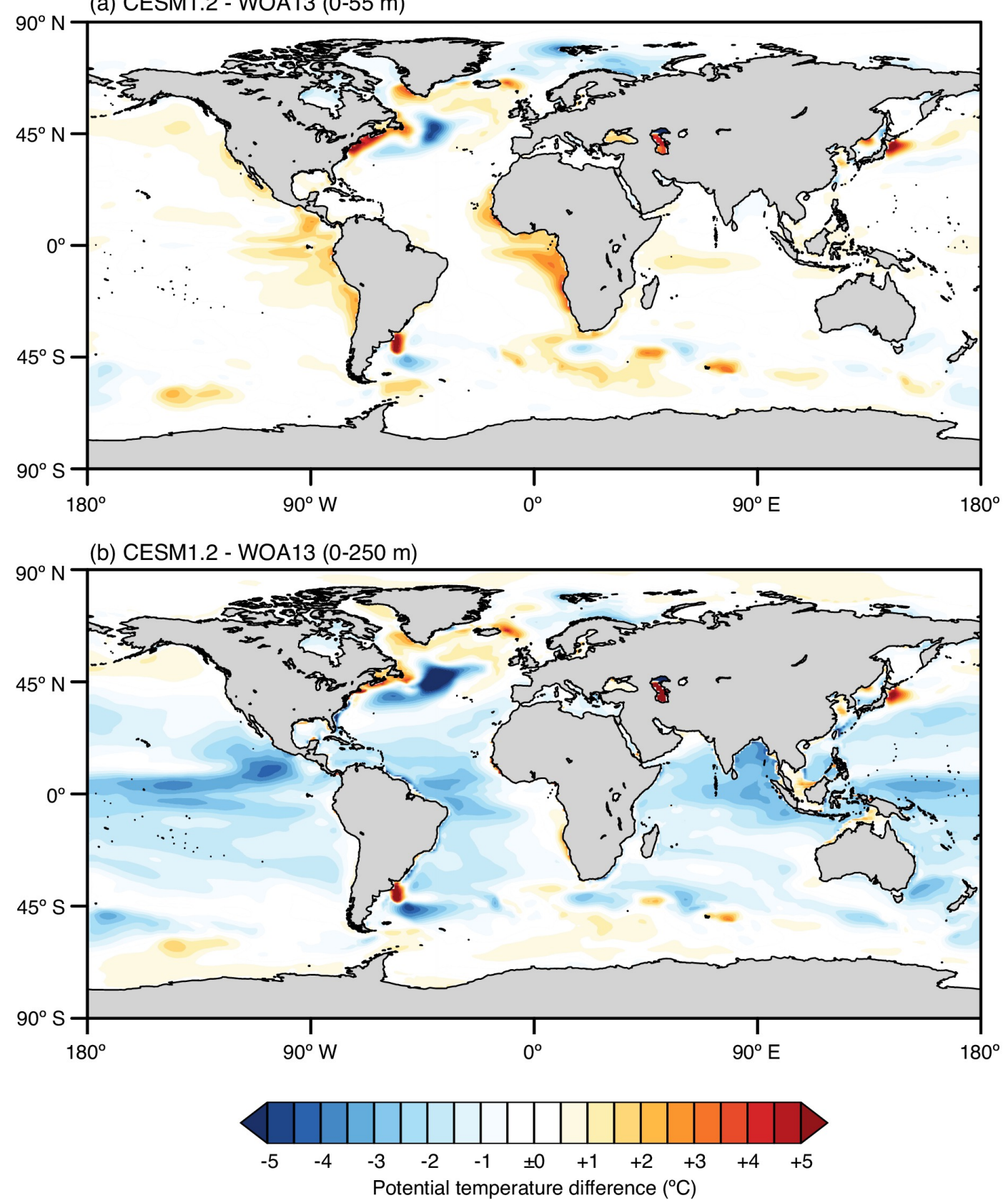
Table S1.

\begin{tabular}{|c|c|c|c|c|c|c|c|c|c|c|}
\hline \# & Site & $\begin{array}{l}\text { Latitude } \\
\left({ }^{\circ} \mathrm{N}\right)\end{array}$ & $\begin{array}{r}\text { Longitude } \\
\left({ }^{\circ} \mathrm{E}\right)\end{array}$ & $\begin{array}{r}\text { Water Depth } \\
(\mathrm{m})\end{array}$ & $\begin{array}{r}\text { Trap Depth } \\
(\mathrm{m})\end{array}$ & $\begin{array}{l}\text { Deployment Time } \\
\text { (day/month/year) }\end{array}$ & $\begin{array}{r}\text { Duration } \\
\text { (days) }\end{array}$ & Species & $\begin{array}{l}\text { Fraction } \\
\qquad(\mu \mathrm{m})\end{array}$ & Source \\
\hline 1 & GS2 & 75.00 & 0.00 & 3720 & 300 & $\begin{array}{l}03 / 06 / 1994 \text { to } \\
11 / 05 / 1995\end{array}$ & 342 & $\mathrm{~Np}$ & $63-500$ & Jensen (1998) \\
\hline 2 & OG5 & 72.40 & -7.70 & 2624 & 500 & $\begin{array}{l}06 / 08 / 1991 \text { to } \\
10 / 07 / 1992\end{array}$ & 339 & $\mathrm{~Np}$ & $63-500$ & Jensen (1998) \\
\hline 3 & NB6/7 & 69.69 & 0.47 & 3273 & 500 & $\begin{array}{l}06 / 08 / 1991 \text { to } \\
02 / 10 / 1993\end{array}$ & 780 & $\mathrm{~Np}, \mathrm{Ni}$ & $63-500$ & Jensen (1998) \\
\hline 4 & PAC50 & 50.01 & 165.03 & 5570 & 3260 & $\begin{array}{l}01 / 12 / 1997 \text { to } \\
10 / 06 / 2001\end{array}$ & 1091 & $\mathrm{~Np}, \mathrm{Ni}, \mathrm{Gb}$ & $>125$ & Kuroyanagi et al. (2002) \\
\hline 5 & PAPA & 50.00 & -145.00 & 4240 & 3800 & $\begin{array}{l}23 / 09 / 1982 \text { to } \\
30 / 08 / 1986\end{array}$ & 1122 & $\mathrm{~Np}, \mathrm{Ni}, \mathrm{Gb}$ & $>125$ & Sautter and Thunell (1989) \\
\hline 6 & SA & 49.00 & -174.00 & 5406 & 4812 & $\begin{array}{l}23 / 08 / 1990 \text { to } \\
03 / 08 / 1999\end{array}$ & 2702 & $\mathrm{~Np}, \mathrm{~Gb}$ & $>125$ & Asahi and Takahashi (2007) \\
\hline 7 & KNOT & 43.97 & 155.06 & 5370 & 2957 & $\begin{array}{l}01 / 12 / 1997 \text { to } \\
12 / 05 / 2000\end{array}$ & 799 & $\begin{array}{l}\mathrm{Np}, \mathrm{Ni}, \mathrm{Gb}, \\
\mathrm{Gr}\end{array}$ & $>125$ & Kuroyanagi et al. (2002) \\
\hline 8 & WCT6 & 42.00 & 155.34 & 5578 & 1091 & $\begin{array}{l}15 / 08 / 1999 \text { to } \\
31 / 08 / 2000\end{array}$ & 382 & $\begin{array}{l}\mathrm{Np}, \mathrm{Ni}, \mathrm{Gb}, \\
\mathrm{Gr}\end{array}$ & $>125$ & Mohiuddin et al. (2005) \\
\hline 9 & WCT2 & 39.00 & 147.00 & $5356-5322$ & $1371 ; 1586$ & $\begin{array}{l}19 / 11 / 1997 \text { to } \\
10 / 08 / 1999\end{array}$ & 608 & $\mathrm{Ni}, \mathrm{Gb}, \mathrm{Gr}$ & $>125$ & Mohiuddin et al. (2002) \\
\hline 10 & WCT7 & 36.68 & 154.94 & 5578 & 5034 & $\begin{array}{l}19 / 08 / 1999 \text { to } \\
29 / 08 / 2000\end{array}$ & 376 & $\mathrm{~Gb}, \mathrm{Ts}$ & $>125$ & Mohiuddin et al. (2004) \\
\hline 11 & WCT1 & 25.00 & 136.99 & $4905-5308$ & $917 ; 1388$ & $\begin{array}{l}07 / 12 / 1997 \text { to } \\
12 / 08 / 1999\end{array}$ & 560 & $\mathrm{Ni}, \mathrm{Gr}, \mathrm{Ts}$ & $>125$ & Mohiuddin et al. (2002) \\
\hline 12 & SBB & 34.23 & -120.03 & 650 & $590 ; 470$ & $\begin{array}{l}12 / 08 / 1993 \text { to } \\
26 / 06 / 1999\end{array}$ & 1015 & $\mathrm{Ni}, \mathrm{Gb}, \mathrm{Gr}$ & $>125$ & $\begin{array}{l}\text { Kincaid et al. (2000) } \\
\text { Darling et al. (2003) }\end{array}$ \\
\hline 13 & SPB & 33.55 & -118.50 & 880 & 500 & $\begin{array}{l}07 / 01 / 1988 \text { to } \\
26 / 07 / 1988\end{array}$ & 199 & $\mathrm{Ni}, \mathrm{Gb}, \mathrm{Gr}$ & $>125$ & Sautter and Thunell (1991) \\
\hline 14 & JGOFS34 & 34.00 & -21.00 & n.a. & 2000 & $\begin{array}{l}03 / 04 / 1989 \text { to } \\
16 / 04 / 1990\end{array}$ & 378 & $\mathrm{Ni}, \mathrm{Gb}, \mathrm{Ts}$ & $>150$ & Wolfteich (1994) \\
\hline 15 & L1 & 33.00 & -22.00 & 5300 & 3000 & $\begin{array}{l}24 / 02 / 2002 \text { to } \\
01 / 04 / 2004\end{array}$ & 764 & $\begin{array}{l}\mathrm{Ni}, \mathrm{Gb}, \mathrm{Gr}, \\
\mathrm{Ts}\end{array}$ & $>125$ & Storz et al. (2009) \\
\hline 16 & BATS & 32.08 & -64.25 & 4200 & 3200 & $\begin{array}{l}06 / 04 / 1978 \text { to } \\
17 / 05 / 1984\end{array}$ & 1848 & $\mathrm{~Gb}, \mathrm{Gr}, \mathrm{Ts}$ & $>125$ & $\begin{array}{l}\text { Deuser et al. (1981) } \\
\text { Deuser and Ross (1989) }\end{array}$ \\
\hline 17 & WAST & 16.32 & 60.47 & 4016 & 3026 & $\begin{array}{l}10 / 05 / 1986 \text { to } \\
21 / 10 / 1987\end{array}$ & 506 & $\mathrm{~Gb}, \mathrm{Gr}, \mathrm{Ts}$ & $>150$ & Curry et al. (1992) \\
\hline 18 & EA1 & 3.17 & -11.25 & 4524 & 984 & $\begin{array}{l}13 / 04 / 1991 \text { to } \\
29 / 11 / 1991\end{array}$ & 230 & $\mathrm{~Gb}, \mathrm{Gr}, \mathrm{Ts}$ & $>150$ & Fischer and Wefer (1996) \\
\hline 19 & EA2 & 1.78 & -11.25 & 4399 & 953 & $\begin{array}{l}13 / 04 / 1991 \text { to } \\
29 / 11 / 1991\end{array}$ & 230 & $\mathrm{~Gb}, \mathrm{Gr}, \mathrm{Ts}$ & $>150$ & Fischer and Wefer (1996) \\
\hline 20 & EA3 & 0.08 & -10.77 & 4141 & 1097 & $\begin{array}{l}13 / 04 / 1991 \text { to } \\
29 / 11 / 1991\end{array}$ & 230 & $\mathrm{~Gb}, \mathrm{Gr}, \mathrm{Ts}$ & $>150$ & Fischer and Wefer (1996) \\
\hline 21 & EA4 & -2.19 & -10.09 & 3906 & 1068 & $\begin{array}{l}\text { 13/04/1991 to } \\
29 / 11 / 1991\end{array}$ & 230 & $\mathrm{~Gb}, \mathrm{Gr}, \mathrm{Ts}$ & $>150$ & Fischer and Wefer (1996) \\
\hline 22 & WA1 & -4.00 & -25.57 & 5530 & 652 & $\begin{array}{l}17 / 10 / 1992 \text { to } \\
21 / 03 / 1993\end{array}$ & 155 & Gr, Ts & $>150$ & Fischer and Wefer (1996) \\
\hline 23 & NCR & -42.70 & 178.63 & 1500 & 1000 & $\begin{array}{l}14 / 09 / 1996 \text { to } \\
15 / 05 / 1997\end{array}$ & 243 & $\begin{array}{l}\mathrm{Np}, \mathrm{Ni}, \mathrm{Gb}, \\
\mathrm{Gr}, \mathrm{Ts}\end{array}$ & $>150$ & King and Howard (2001) \\
\hline 24 & SCR & -44.62 & 178.62 & 1500 & 1000 & $\begin{array}{l}09 / 06 / 1996 \text { to } \\
15 / 05 / 1997\end{array}$ & 340 & $\begin{array}{l}\mathrm{Np}, \mathrm{Ni}, \mathrm{Gb}, \\
\mathrm{Gr}, \mathrm{Ts}\end{array}$ & $>150$ & King and Howard (2001) \\
\hline 25 & $\mathrm{CP}$ & -52.62 & 174.15 & n.a. & $442 ; 362$ & $\begin{array}{l}14 / 05 / 1998 \text { to } \\
13 / 07 / 1999\end{array}$ & 368 & $\mathrm{~Np}, \mathrm{Ni}, \mathrm{Gb}$ & $>150$ & Northcote and Neil (2005) \\
\hline 26 & WS34 & -64.90 & -2.60 & 5053 & 360 & $\begin{array}{l}16 / 01 / 1988 \text { to } \\
26 / 02 / 1990\end{array}$ & 745 & $\mathrm{~Np}$ & $>125$ & Donner and Wefer (1994) \\
\hline
\end{tabular}

$\mathrm{Np}-N$. pachyderma; $\mathrm{Ni}-N$. incompta; $\mathrm{Gb}-$ G. bulloides; $\mathrm{Gr}-$ G. ruber (white); $\mathrm{Ts}-T$. sacculifer

n.a. - not available (i.e., not given in data set) 
Table S2.

\begin{tabular}{|c|c|c|c|c|c|c|c|c|c|}
\hline$\#$ & Station & $\begin{array}{r}\text { Latitude } \\
\left({ }^{\circ} \mathrm{N}\right)\end{array}$ & $\begin{array}{r}\text { Longitude } \\
\left({ }^{\circ} \mathrm{E}\right)\end{array}$ & $\begin{array}{r}\text { Water Depth } \\
(\mathrm{m})\end{array}$ & Depth Intervals & $\begin{array}{c}\text { Date } \\
\text { (season) }\end{array}$ & Species & $\begin{array}{l}\text { Fraction } \\
(\mu \mathrm{m})\end{array}$ & Source \\
\hline 1 & $93-36$ & 80.36 & -10.14 & n.a. & $\begin{array}{l}0-20,20-40,40-60,60-100 \\
100-150,150-200,200-250 \\
250-275\end{array}$ & $\begin{array}{l}27 / 07 / 1993 \\
\text { (summer) }\end{array}$ & $\mathrm{Np}$ & n.a. & Kohfeld et al. (1996) \\
\hline 2 & PS78-25 & 78.83 & 7.00 & 1465 & $\begin{array}{l}0-50,50-100,100-200 \\
200-300,300-500\end{array}$ & $\begin{array}{l}26 / 06 / 2011 \\
\text { (summer) }\end{array}$ & $\mathrm{Np}, \mathrm{Ni}, \mathrm{Gb}$ & $100-250$ & Pados and Spielhagen (2014) \\
\hline 3 & PS78-44 & 78.83 & 0.08 & 2636 & $\begin{array}{l}0-50,50-100,100-200, \\
200-300,300-500\end{array}$ & $\begin{array}{l}\text { 29/06/2011 } \\
\text { (summer) }\end{array}$ & $\mathrm{Np}, \mathrm{Ni}, \mathrm{Gb}$ & $100-250$ & Pados and Spielhagen (2014) \\
\hline 4 & PS78-75 & 78.83 & -3.92 & 1978 & $\begin{array}{l}0-50,50-100,100-200 \\
200-300,300-500\end{array}$ & $\begin{array}{l}04 / 07 / 2011 \\
\text { (summer) }\end{array}$ & $\mathrm{Np}, \mathrm{Ni}, \mathrm{Gb}$ & $100-250$ & Pados and Spielhagen (2014) \\
\hline 5 & PS55-025 & 75.00 & -10.58 & 3084 & $\begin{array}{l}0-50,50-100,100-200 \\
200-300,300-500\end{array}$ & $\begin{array}{l}11 / 07 / 1999 \\
\text { (summer) }\end{array}$ & $\mathrm{Np}, \mathrm{Ni}, \mathrm{Gb}$ & $125-250$ & Stangeew (2001) \\
\hline 6 & PS55-043 & 75.00 & 0.36 & 3789 & $\begin{array}{l}0-50,50-100,100-200 \\
200-300,300-500\end{array}$ & $\begin{array}{l}14 / 07 / 1999 \\
\text { (summer) }\end{array}$ & $\mathrm{Np}, \mathrm{Ni}, \mathrm{Gb}$ & $125-250$ & Stangeew (2001) \\
\hline 7 & PS55-063 & 75.00 & 10.65 & 2542 & $\begin{array}{l}0-50,50-100,100-200 \\
200-300,300-500\end{array}$ & $\begin{array}{l}16 / 07 / 1999 \\
\text { (summer) }\end{array}$ & $\mathrm{Np}, \mathrm{Ni}, \mathrm{Gb}$ & $125-250$ & Stangeew (2001) \\
\hline 8 & MN116 & 75.00 & -7.31 & 3393 & $\begin{array}{l}0-50,50-100,100-150, \\
150-300\end{array}$ & $\begin{array}{l}21 / 08 / 1994 \\
\text { (summer) }\end{array}$ & $\mathrm{Np}$ & $125-250$ & Simstich et al. (2003) \\
\hline 9 & MN2 & 70.00 & 3.40 & 3261 & $\begin{array}{l}0-50,50-100,100-500 \\
500-1000,1000-2000\end{array}$ & $\begin{array}{l}10 / 07 / 1994 \\
\text { (summer) }\end{array}$ & $\mathrm{Np}$ & $125-250$ & Simstich et al. (2003) \\
\hline 10 & MN323 & 69.69 & 0.47 & 3290 & $\begin{array}{l}0-20,20-40,40-60,60-80 \\
80-100,100-200,200-300 \\
300-500,500-700,700-1000 \\
1000-1500,1500-2000,2000-2500\end{array}$ & $\begin{array}{l}07 / 07 / 1992 \\
\text { (summer) }\end{array}$ & $\mathrm{Np}$ & $125-250$ & Simstich et al. (2003) \\
\hline 11 & MN314 & 67.54 & 5.58 & 1438 & $\begin{array}{l}0-20,20-40,40-60,60-80 \\
80-100,100-200,200-300 \\
300-500,500-700\end{array}$ & $\begin{array}{l}\text { 28/06/1992 } \\
\text { (summer) }\end{array}$ & $\mathrm{Np}$ & $125-250$ & Simstich et al. (2003) \\
\hline 12 & PAPA & 49.98 & -144.97 & 4253 & $\begin{array}{l}0-50,50-100,100-150 \\
150-200,200-300\end{array}$ & $\begin{array}{l}16 / 08 / 2015 \\
\text { (summer) }\end{array}$ & $\mathrm{Np}, \mathrm{Ni}, \mathrm{Gb}$ & $>100$ & Iwasaki et al. (2017) \\
\hline 13 & 101 & 47.00 & -174.95 & 5790 & $\begin{array}{l}0-50,50-100,100-150 \\
150-200,200-300\end{array}$ & $\begin{array}{c}05 / 08 / 2015 \\
\text { (summer) }\end{array}$ & $\mathrm{Np}, \mathrm{Ni}, \mathrm{Gb}$ & $>100$ & Iwasaki et al. (2017) \\
\hline 14 & 79 & 46.98 & 166.73 & 5957 & $\begin{array}{l}0-50,50-100,100-150, \\
150-200,200-300\end{array}$ & $\begin{array}{l}31 / 07 / 2015 \\
\text { (summer) }\end{array}$ & $\mathrm{Np}, \mathrm{Ni}, \mathrm{Gb}$ & $>100$ & Iwasaki et al. (2017) \\
\hline 15 & KNOT & 44.08 & 154.98 & 5335 & $\begin{array}{l}0-50,50-100,100-150 \\
150-200,200-300\end{array}$ & $\begin{array}{l}25 / 07 / 2015 \\
\text { (summer) }\end{array}$ & $\mathrm{Np}, \mathrm{Ni}, \mathrm{Gb}$ & $>100$ & Iwasaki et al. (2017) \\
\hline 16 & $\# \mathrm{~B}$ & 41.57 & 141.90 & 1000 & $\begin{array}{l}0-20,20-40,40-60,60-80 \\
80-120,120-160,160-200\end{array}$ & $\begin{array}{l}03 / 06 / 2002 \\
\text { (summer) }\end{array}$ & $\begin{array}{l}\mathrm{Np}, \mathrm{Ni}, \mathrm{Gb} \\
\mathrm{Gr}, \mathrm{Ts}\end{array}$ & $125-1000$ & Kuroyanagi and Kawahata (2004) \\
\hline 17 & $\# \mathrm{~b}$ & 41.15 & 143.38 & 2077 & $\begin{array}{l}0-20,20-40,40-60,60-80 \\
80-120,120-160,160-200\end{array}$ & $\begin{array}{c}04 / 06 / 2002 \\
\text { (summer) }\end{array}$ & $\begin{array}{l}\mathrm{Np}, \mathrm{Ni}, \mathrm{Gb} \\
\mathrm{Gr}, \mathrm{Ts}\end{array}$ & $125-1000$ & Kuroyanagi and Kawahata (2004) \\
\hline 18 & \#A & 36.02 & 141.78 & 2220 & $\begin{array}{l}0-20,20-40,40-60,60-80 \\
80-120,120-160,160-200\end{array}$ & $\begin{array}{l}\text { 25/05/2002 } \\
\text { (spring) }\end{array}$ & $\begin{array}{l}\mathrm{Np}, \mathrm{Ni}, \mathrm{Gb} \\
\mathrm{Gr}, \mathrm{Ts}\end{array}$ & $125-1000$ & Kuroyanagi and Kawahata (2004) \\
\hline 19 & $\# \mathrm{E}$ & 32.17 & 133.88 & 2660 & $\begin{array}{l}0-20,20-40,40-60,60-80 \\
80-120,120-160,160-200\end{array}$ & $\begin{array}{l}27 / 05 / 2002 \\
\text { (spring) }\end{array}$ & $\begin{array}{l}\mathrm{Np}, \mathrm{Ni}, \mathrm{Gb}, \\
\mathrm{Gr}, \mathrm{Ts}\end{array}$ & $125-1000$ & Kuroyanagi and Kawahata (2004) \\
\hline 20 & POS383-165 & 34.00 & -22.00 & 5288 & $\begin{array}{l}0-100,100-200,200-300 \\
300-500,500-700\end{array}$ & $\begin{array}{l}23 / 04 / 2009 \\
\text { (spring) }\end{array}$ & $\begin{array}{l}\mathrm{Np}, \mathrm{Ni}, \mathrm{Gb}, \\
\mathrm{Gr}, \mathrm{Ts}\end{array}$ & $>100$ & Rebotim et al. (2017) \\
\hline 21 & POS $383-175$ & 33.15 & -22.00 & 5232 & $\begin{array}{l}0-100,100-200,200-300 \\
300-500,500-700\end{array}$ & $\begin{array}{l}\text { 26/04/2009 } \\
\text { (spring) }\end{array}$ & $\begin{array}{l}\mathrm{Np}, \mathrm{Ni}, \mathrm{Gb}, \\
\mathrm{Gr}, \mathrm{Ts}\end{array}$ & $>100$ & Rebotim et al. (2017) \\
\hline 22 & POS247-1389 & 33.08 & -22.00 & 5226 & $\begin{array}{l}0-20,20-40,40-60,60-80 \\
80-100,100-200,200-300 \\
300-500,500-700\end{array}$ & $\begin{array}{l}\text { 24/01/1999 } \\
\text { (winter) }\end{array}$ & $\begin{array}{l}\text { Np, Ni, Gb, } \\
\text { Gr, Ts }\end{array}$ & $>100$ & Rebotim et al. (2017) \\
\hline 23 & MOC1-38 & 38.92 & -67.90 & n.a. & $\begin{array}{l}0-25,25-50,50-75,75-100 \\
100-125,125-150,150-175 \\
175-200\end{array}$ & $\begin{array}{l}11 / 1975 \\
\text { (fall) }\end{array}$ & $\mathrm{Gb}, \mathrm{Gr}, \mathrm{Ts}$ & n.a. & Fairbanks et al. (1980) \\
\hline 24 & MOC1-28 & 33.91 & -71.78 & n.a. & $\begin{array}{l}0-25,25-50,50-75,75-100 \\
100-125,125-150,150-175 \\
175-200\end{array}$ & $\begin{array}{l}11 / 1975 \\
\quad(\text { fall })\end{array}$ & Gr, Ts & n.a. & Fairbanks et al. (1980) \\
\hline 25 & MOC1-23 & 32.73 & -71.16 & n.a. & $\begin{array}{l}0-25,25-50,50-75,75-100 \\
100-125,125-150,150-175 \\
175-200\end{array}$ & $\begin{array}{c}11 / 1975 \\
\text { (fall) }\end{array}$ & $\mathrm{Gr}, \mathrm{Ts}$ & n.a. & Fairbanks et al. (1980) \\
\hline 26 & 310 & 16.02 & 52.73 & n.a. & $\begin{array}{l}0-10,10-25,25-50,50-75 \\
75-100,100-150,150-200 \\
200-300,300-500\end{array}$ & $\begin{array}{c}\text { 20/08/1992 } \\
\text { (summer) }\end{array}$ & $\begin{array}{l}\mathrm{Np}, \mathrm{Gb}, \mathrm{Gr} \\
\mathrm{Ts}\end{array}$ & $>125$ & Peeters and Brummer (2002) \\
\hline 27 & 920 & 16.09 & 52.70 & n.a. & $\begin{array}{l}0-10,10-25,25-50,50-75, \\
75-100,100-150,150-200, \\
200-300,300-500\end{array}$ & $\begin{array}{l}\text { 27/02/1993 } \\
\text { (winter) }\end{array}$ & $\begin{array}{l}\text { Np, Gb, Gr, } \\
\text { Ts }\end{array}$ & $>125$ & Peeters and Brummer (2002) \\
\hline 28 & 313 & 15.91 & 53.02 & n.a. & $\begin{array}{l}0-10,10-25,25-50,50-75, \\
75-100,100-150,150-200, \\
200-300,300-500\end{array}$ & $\begin{array}{l}21 / 08 / 1992 \\
\text { (summer) }\end{array}$ & $\begin{array}{l}\text { Np, Gb, Gr, } \\
\text { Ts }\end{array}$ & $>125$ & Peeters and Brummer (2002) \\
\hline 29 & 917 & 15.89 & 52.97 & n.a. & $\begin{array}{l}0-10,10-25,25-50,50-75 \\
75-100,100-150,150-200 \\
200-300,300-500\end{array}$ & $\begin{array}{l}\text { 25/02/1993 } \\
\text { (winter) }\end{array}$ & $\begin{array}{l}\text { Np, Gb, Gr, } \\
\text { Ts }\end{array}$ & $>125$ & Peeters and Brummer (2002) \\
\hline 30 & MOC63 & 2.92 & -140.20 & n.a. & $0-10,10-20,20-40,40-60$ & 22/08/1992 & $\mathrm{Gb}, \mathrm{Gr}, \mathrm{Ts}$ & $>150$ & Watkins et al. (1998) \\
\hline
\end{tabular}




\begin{tabular}{|c|c|c|c|c|c|c|c|c|c|}
\hline$\#$ & Station & $\begin{array}{r}\text { Latitude } \\
\left({ }^{\circ} \mathrm{N}\right)\end{array}$ & $\begin{array}{r}\text { Longitude } \\
\left({ }^{\circ} \mathrm{E}\right)\end{array}$ & $\begin{array}{r}\text { Water Depth } \\
(\mathrm{m})\end{array}$ & Depth Intervals & $\begin{array}{c}\text { Date } \\
\text { (season) }\end{array}$ & Species & $\begin{array}{r}\text { Fraction } \\
(\mu \mathrm{m})\end{array}$ & Source \\
\hline & & & & & $\begin{array}{l}60-80,80-100,100-150, \\
150-200\end{array}$ & (summer) & & & \\
\hline 31 & MOC65 & 2.05 & -141.49 & n.a. & $\begin{array}{l}10-20,40-60,60-80 \\
80-100,100-150,150-200\end{array}$ & $\begin{array}{c}26 / 08 / 1992 \\
\text { (summer) }\end{array}$ & $\mathrm{Gb}, \mathrm{Gr}, \mathrm{Ts}$ & $>150$ & Watkins et al. (1998) \\
\hline 32 & MOC12 & 2.01 & -139.88 & n.a. & $\begin{array}{l}0-5,5-10,10-30,30-50,50-70 \\
70-90,90-145,145-200\end{array}$ & $\begin{array}{l}17 / 02 / 1992 \\
\text { (winter) }\end{array}$ & $\mathrm{Gb}, \mathrm{Gr}, \mathrm{Ts}$ & $>150$ & Watkins et al. (1996) \\
\hline 33 & MOC66 & 1.13 & -140.01 & n.a. & $\begin{array}{l}0-10,10-20,20-40,40-60 \\
60-80,80-100,100-150 \\
150-200\end{array}$ & $\begin{array}{c}27 / 08 / 1992 \\
\text { (summer) }\end{array}$ & $\mathrm{Gb}, \mathrm{Gr}, \mathrm{Ts}$ & $>150$ & Watkins et al. (1998) \\
\hline 34 & MOC15 & 0.00 & -140.07 & n.a. & $\begin{array}{l}0-10,10-20,20-40,40-60 \\
60-100,100-150\end{array}$ & $\begin{array}{l}\text { 23/02/1992 } \\
\text { (winter) }\end{array}$ & Gr, Ts & $>150$ & Watkins et al. (1996) \\
\hline 35 & MOC69 & -1.05 & -139.97 & n.a. & $\begin{array}{l}0-10,10-20,20-40,40-60 \text {, } \\
60-80,100-150,150-200\end{array}$ & $\begin{array}{l}01 / 09 / 1992 \\
(\text { fall })\end{array}$ & $\mathrm{Gb}, \mathrm{Gr}, \mathrm{Ts}$ & $>150$ & Watkins et al. (1998) \\
\hline 36 & MOC20 & -2.02 & -140.16 & n.a. & $\begin{array}{l}0-10,10-20,20-40,40-60 \\
60-80,80-100,100-150 \\
150-200\end{array}$ & $\begin{array}{l}\text { 29/02/1992 } \\
\text { (winter) }\end{array}$ & Gr, Ts & $>150$ & Watkins et al. (1996) \\
\hline 37 & MOC71 & -2.33 & -140.32 & n.a. & $\begin{array}{l}0-10,10-20,20-40,40-60, \\
60-80,80-100,100-150, \\
150-200\end{array}$ & $\begin{array}{l}\text { 04/09/1992 } \\
\text { (fall) }\end{array}$ & $\mathrm{Gb}, \mathrm{Gr}, \mathrm{Ts}$ & $>150$ & Watkins et al. (1998) \\
\hline 38 & MOC72 & -3.21 & -140.25 & n.a. & $\begin{array}{l}0-10,10-20,20-40,40-60, \\
60-80,80-100,100-150, \\
150-200\end{array}$ & $\begin{array}{l}\text { 06/09/1992 } \\
\text { (fall) }\end{array}$ & $\mathrm{Gb}, \mathrm{Gr}, \mathrm{Ts}$ & $>150$ & Watkins et al. (1998) \\
\hline 39 & SO225-21-3 & -3.05 & -165.06 & 5188 & $\begin{array}{l}0-50,50-100,100-200 \\
200-300,300-500\end{array}$ & $\begin{array}{c}08 / 12 / 2012 \\
\text { (winter) }\end{array}$ & $\mathrm{Gb}, \mathrm{Gr}, \mathrm{Ts}$ & $>150$ & Rippert et al. (2016) \\
\hline 40 & TNO57-16 & -50.12 & 5.75 & 3761 & $\begin{array}{l}0-30,30-60,60-75,75-85 \\
85-125,125-235,235-300 \\
300-440\end{array}$ & $\begin{array}{l}\text { 24/02/1996 } \\
\text { (winter) }\end{array}$ & $\mathrm{Np}, \mathrm{Ni}, \mathrm{Gb}$ & $>150$ & Mortyn and Charles (2003) \\
\hline 41 & TNO57-13 & -53.18 & 5.13 & 2851 & $\begin{array}{l}0-25,25-50,50-75,75-100 \\
100-150,150-200,200-300 \\
300-400,400-500\end{array}$ & $\begin{array}{l}\text { 21/02/1996 } \\
\text { (winter) }\end{array}$ & $\mathrm{Np}, \mathrm{Ni}, \mathrm{Gb}$ & $>150$ & Mortyn and Charles (2003) \\
\hline 42 & AN98/O & -63.25 & 177.25 & 4100 & $0-50,50-90,90-130$ & $\begin{array}{l}\text { 20/01/1998 } \\
\text { (winter) }\end{array}$ & $\mathrm{Np}, \mathrm{Ni}, \mathrm{Gb}$ & $>100$ & Bergami et al. (2009) \\
\hline 43 & AN99/O & -63.40 & 178.05 & 4074 & $0-35,35-70$ & $\begin{array}{l}09 / 01 / 1999 \\
\text { (winter) }\end{array}$ & $\mathrm{Np}, \mathrm{Ni}, \mathrm{Gb}$ & $>100$ & Bergami et al. (2009) \\
\hline 44 & ANO0/O & -63.53 & 178.38 & 3548 & $0-60,60-150,150-220$ & $\begin{array}{l}11 / 01 / 2000 \\
\text { (winter) }\end{array}$ & $\mathrm{Np}, \mathrm{Ni}, \mathrm{Gb}$ & $>100$ & Bergami et al. (2009) \\
\hline 45 & AN01/O & -63.43 & 178.10 & 3964 & $0-90,90-150$ & $\begin{array}{l}09 / 01 / 2001 \\
\text { (winter) }\end{array}$ & $\mathrm{Np}, \mathrm{Ni}, \mathrm{Gb}$ & $>100$ & Bergami et al. (2009) \\
\hline
\end{tabular}

$\mathrm{Np}-N$. pachyderma; $\mathrm{Ni}-N$. incompta $; \mathrm{Gb}-$ G. bulloides; $\mathrm{Gr}-$ G. ruber (white); $\mathrm{Ts}-$ T. sacculifer n.a. - not available (i.e., not given in data set)

Here the season refers to those of the Northern Hemisphere. 
Table S3a.

\begin{tabular}{|c|c|c|c|c|c|c|c|c|c|c|c|c|c|}
\hline \multirow[t]{2}{*}{ Province } & \multicolumn{3}{|c|}{ Sediment Trap Details } & \multicolumn{2}{|c|}{ N. pachyderma } & \multicolumn{2}{|c|}{ N. incompta } & \multicolumn{2}{|c|}{ G. bulloides } & \multicolumn{2}{|c|}{ G. ruber (white) } & \multicolumn{2}{|c|}{ T. sacculifer } \\
\hline & Site & $\begin{array}{r}\text { Latitude } \\
\left({ }^{\circ} \mathrm{N}\right)\end{array}$ & $\begin{array}{r}\text { Longitude } \\
\left({ }^{\circ} \mathrm{E}\right)\end{array}$ & Trap & PLAFOM2.0 & Trap & PLAFOM2.0 & Trap & PLAFOM2.0 & Trap & PLAFOM2.0 & Trap & PLAFOM2.0 \\
\hline \multirow{3}{*}{$\frac{\text { 훙 }}{2}$} & GS2 & 75.00 & 0.00 & Jun-Sep & Jul-Sep & - & - & - & - & - & - & - & - \\
\hline & OG5 & 72.40 & -7.70 & Aug-Sep & Jul-Sep & - & - & - & - & - & - & - & - \\
\hline & NB6/7 & 69.69 & -0.47 & Jun-Nov & Apr-Jun & Jul-Nov & Jul-Oct & - & - & - & - & - & - \\
\hline \multirow{3}{*}{$\begin{array}{l}\frac{7}{0} \\
0 \\
0 \\
0 \\
\tilde{n}\end{array}$} & PAC50 & 50.01 & 165.03 & \multirow{3}{*}{$\begin{array}{c}\text { Mar-May } \\
\text { Sep-Nov } \\
\text { Mar-May } \\
\text { Nov-Dec } \\
\text { Apr-Jul } \\
\text { Aug-Oct }\end{array}$} & \multirow{2}{*}{$\begin{array}{l}\text { Feb-May } \\
\text { Jul-Sep } \\
\text { Mar-May }\end{array}$} & \multirow{2}{*}{$\begin{array}{l}\text { Mar-May } \\
\text { Apr-Jun }\end{array}$} & \multirow{2}{*}{$\begin{array}{l}\text { Aug-Nov } \\
\text { Aug-Nov }\end{array}$} & \multirow{3}{*}{$\begin{array}{c}\text { Apr-Jun } \\
\\
\text { Apr-Jun } \\
\text { Nov-Dec } \\
\text { Apr-May } \\
\text { Jul-Sep }\end{array}$} & Aug-Nov & - & - & - & - \\
\hline & PAPA & 50.00 & -145.00 & & & & & & Apr-May & - & - & - & - \\
\hline & SA & 49.00 & -174.00 & & Mar-May & - & - & & Apr-Jun & - & - & - & - \\
\hline \multirow{4}{*}{ 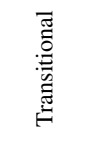 } & KNOT & 43.97 & 155.06 & Mar-May & Mar-May & none & Jul-Oct & Mar-May & Apr-Jun & Sep-Nov & Aug-Oct & - & - \\
\hline & WCT6 & 42.00 & 155.34 & \multirow{3}{*}{$\begin{array}{c}\text { Mar-May } \\
\text { Jul-Sep } \\
- \\
-\end{array}$} & Feb-May & Jun-Oct & Mar-May & Mar-May & Mar-May & Sep-Nov & Aug-Oct & - & - \\
\hline & WCT2 & 39.00 & 147.00 & & - & none & Jan-Apr & none & Jan-Apr & Sep-Dec & Jul-Oct & - & - \\
\hline & WCT7 & 36.68 & 154.94 & & - & 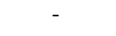 & - & May-Jun & Mar-May & & 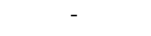 & Aug-Dec & Aug-Nov \\
\hline & $\mathrm{SBB}^{\mathrm{a}}$ & 34.23 & -120.03 & - & - & Aug-Oct & Jul-Sep & Jun-Aug & Jul-Sep & Jul-Nov & Aug-Nov & - & - \\
\hline .3 & JGOFS34 & 34.00 & -21.00 & - & - & $\begin{array}{l}\text { Feb-Apr } \\
\text { Aug-Sep }\end{array}$ & May-Aug & Mar-May & May-Sep & 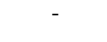 & 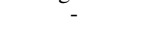 & Apr-Jun & Jul-Oct \\
\hline ర్రి & SPB & 33.55 & -118.50 & - & - & Mar-May & May-Sep & May-Jun & May-Sep & Apr-Jul & Aug-Nov & - & - \\
\hline 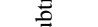 & $\mathrm{L} 1$ & 33.00 & -22.00 & - & - & Feb-Apr & May-Sep & Mar-May & May-Sep & Mar-May & Jul-Nov & Mar-May & Jul-Oct \\
\hline 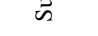 & BATS & 32.08 & -64.25 & - & - & - & - & Jan-May & Feb & none & May-Jul & May-Jul & Jul-Nov \\
\hline & WCT1 & 25.00 & 136.99 & - & - & none & Feb-Jun & - & $\begin{array}{c}\text { Mray-sep } \\
-\end{array}$ & Jun-Aug & Jan-Mar & Jun-Aug & Jun-Oct \\
\hline & WAST & 16.32 & 60.47 & - & - & - & - & $\begin{array}{l}\text { Jan-Feb } \\
\text { Jul-Oct }\end{array}$ & $\begin{array}{l}\text { Mar-May } \\
\text { Oct-Nov }\end{array}$ & $\begin{array}{l}\text { Dec-Feb } \\
\text { May-Aug }\end{array}$ & $\begin{array}{l}\text { Jan-Mar } \\
\text { Aug-Sep }\end{array}$ & Jun-Sep & Apr-May \\
\hline & EA1 & 3.17 & -11.25 & - & - & - & - & Sep-Nov & Feb-Apr & none & Jul-Sep & none & Jun-Aug \\
\hline$\frac{0}{2}$ & EA2 & 1.78 & -11.25 & - & - & - & - & Jul-Oct & $\begin{array}{l}\text { Mar-Apr } \\
\text { Aug_Sep }\end{array}$ & none & Jul-Nov & May-Jul & $\begin{array}{l}\text { Jan-Feb } \\
\text { Sep-Noy }\end{array}$ \\
\hline$\oplus$ & EA3 & 0.08 & -10.77 & - & - & - & - & Jul-Sep & Feb-Apr & May-Jun & Sep-Jan & May-Jun & Feb-May \\
\hline & EA4 & -2.19 & -10.09 & - & - & - & - & Apr-May & Feb-Apr & Apr-Aug & Aug-Dec & Apr-Aug & Dec-May \\
\hline & WA1 & -4.00 & -25.57 & - & - & - & - & . & 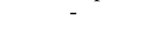 & none & Aug-Dec & none & Aug-Dec \\
\hline 胥 & NCR & -42.70 & 178.63 & Sep-Dec & $\begin{array}{l}\text { Apr-May } \\
\text { Aug-Nov }\end{array}$ & Sep-Oct & $\begin{array}{l}\text { Jan-Feb } \\
\text { Aug-Oct }\end{array}$ & $\begin{array}{l}\text { Apr-May } \\
\text { Sep-Nov }\end{array}$ & Aug-Oct & none & Jan-Apr & Oct-Dec & Jan-Apr \\
\hline 晃: & SCR & -44.62 & 178.62 & Sep-Oct & Aug-Oct & $\begin{array}{l}\text { Feb-Apr } \\
\text { Sep-Nov }\end{array}$ & $\begin{array}{l}\text { Aug-Sep } \\
\text { Dec-Feb }\end{array}$ & Sep-Oct & $\begin{array}{l}\text { Aug-Oct } \\
\text { Dec-Jan }\end{array}$ & - & - & - & - \\
\hline 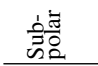 & $\mathrm{CP}$ & -52.62 & 174.15 & Mar-May & Aug-Nov & Mar-May & $\begin{array}{l}\text { Feb-Apr } \\
\text { Aug-Oct }\end{array}$ & Nov-May & $\begin{array}{l}\text { Feb-Mar } \\
\text { Aug-Oct }\end{array}$ & - & - & - & - \\
\hline 흉 & WS34 & -64.90 & -2.60 & Mar-May & $\begin{array}{l}\text { Feb-Apr } \\
\text { Oct-Dec }\end{array}$ & - & - & - & - & - & - & - & - \\
\hline
\end{tabular}

${ }^{a}$ The nearest model grid point for site SBB fell onto land. Therefore, we used the nearest model grid point in the ocean to perform a consistent model-data-comparison. 
Table S3b.

\begin{tabular}{|c|c|c|c|c|c|c|c|c|c|c|c|c|c|}
\hline \multirow[t]{2}{*}{ Province } & \multicolumn{3}{|c|}{ Sediment Trap Details } & \multicolumn{2}{|c|}{ N. pachyderma } & \multicolumn{2}{|c|}{ N. incompta } & \multicolumn{2}{|c|}{ G. bulloides } & \multicolumn{2}{|c|}{ G. ruber (white) } & \multicolumn{2}{|c|}{ T. sacculifer } \\
\hline & Site & $\begin{array}{r}\text { Latitude } \\
\left({ }^{\circ} \mathrm{N}\right)\end{array}$ & $\begin{array}{r}\text { Longitude } \\
\left({ }^{\circ} \mathrm{E}\right)\end{array}$ & Trap & PLAFOM2.0 & Trap & PLAFOM2.0 & Trap & PLAFOM2.0 & Trap & PLAFOM2.0 & Trap & PLAFOM2.0 \\
\hline \multirow{3}{*}{$\frac{\overline{3}}{0}$} & GS2 & 75.00 & 0.00 & 0.78 & 0.26 & - & - & - & - & - & - & - & - \\
\hline & OG5 & 72.40 & -7.70 & 0.64 & 0.19 & - & - & - & - & - & - & - & - \\
\hline & NB6/7 & 69.69 & -0.47 & 0.80 & 0.42 & 0.95 & 0.57 & - & - & - & - & - & - \\
\hline \multirow{3}{*}{ 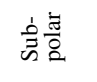 } & PAC50 & 50.01 & 165.03 & 0.75 & 0.20 & 0.77 & 0.36 & 0.66 & 0.36 & - & - & - & - \\
\hline & PAPA & 50.00 & -145.00 & 1.07 & 0.28 & 1.20 & 0.04 & 1.10 & 0.22 & - & - & - & - \\
\hline & SA & 49.00 & -174.00 & 0.95 & 0.29 & - & - & 0.94 & 0.21 & - & - & - & - \\
\hline \multirow{4}{*}{ 竘怘 } & KNOT & 43.97 & 155.06 & 0.69 & 0.36 & 0.79 & 0.16 & 0.66 & 0.33 & 0.76 & 0.70 & - & - \\
\hline & WCT6 & 42.00 & 155.34 & 0.42 & 0.33 & 0.47 & 0.15 & 0.62 & 0.25 & 0.73 & 0.59 & - & - \\
\hline & WCT2 & 39.00 & 147.00 & - & - & 0.64 & 0.17 & 0.69 & 0.22 & 0.74 & 0.44 & - & - \\
\hline & WCT7 & 36.68 & 154.94 & - & - & - & - & 0.55 & 0.19 & - & - & 0.57 & 0.34 \\
\hline \multirow{6}{*}{ 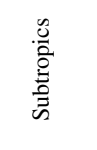 } & $\mathrm{SBB}^{\mathrm{a}}$ & 34.23 & -120.03 & - & - & 0.68 & 0.04 & 0.67 & 0.06 & 0.87 & 0.19 & - & - \\
\hline & JGOFS34 & 34.00 & -21.00 & - & - & 0.85 & 0.05 & 0.76 & 0.07 & - & - & 0.69 & 0.14 \\
\hline & SPB & 33.55 & -118.50 & - & - & 0.73 & 0.04 & 0.85 & 0.06 & 0.77 & 0.18 & - & - \\
\hline & $\mathrm{L} 1$ & 33.00 & -22.00 & - & - & 1.28 & 0.05 & 0.91 & 0.06 & 0.70 & 0.09 & 0.59 & 0.13 \\
\hline & BATS & 32.08 & -64.25 & - & - & - & - & 0.72 & 0.05 & 0.37 & 0.07 & 0.96 & 0.12 \\
\hline & WCT1 & 25.00 & 136.99 & - & - & 0.42 & 0.06 & - & - & 0.77 & 0.10 & 0.88 & 0.07 \\
\hline \multirow{6}{*}{ 嶑 } & WAST & 16.32 & 60.47 & - & - & - & - & 0.77 & 0.02 & 0.70 & 0.14 & 0.66 & 0.02 \\
\hline & EA1 & 3.17 & -11.25 & - & - & - & - & 0.48 & 0.03 & 0.36 & 0.15 & 0.47 & 0.07 \\
\hline & EA2 & 1.78 & -11.25 & - & - & - & - & 0.52 & 0.02 & 0.33 & 0.13 & 0.59 & 0.03 \\
\hline & EA3 & 0.08 & -10.77 & - & - & - & - & 0.81 & 0.02 & 0.57 & 0.10 & 0.47 & 0.05 \\
\hline & EA4 & -2.19 & -10.09 & - & - & - & - & 0.83 & 0.02 & 0.60 & 0.10 & 0.50 & 0.03 \\
\hline & WA1 & -4.00 & -25.57 & - & - & - & - & - & - & 0.67 & 0.15 & 0.58 & 0.06 \\
\hline \multirow{2}{*}{ 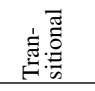 } & NCR & -42.70 & 178.63 & 0.91 & 0.47 & 0.78 & 0.11 & 0.73 & 0.16 & 0.77 & 0.46 & 0.85 & 0.54 \\
\hline & SCR & -44.62 & 178.62 & 0.87 & 0.14 & 0.57 & 0.08 & 0.89 & 0.16 & - & - & - & - \\
\hline 尚㐘 & $\mathrm{CP}$ & -52.62 & 174.15 & 1.29 & 0.28 & 1.12 & 0.08 & 1.10 & 0.11 & - & - & - & - \\
\hline$\frac{\hbar}{0}$ & WS34 & -64.90 & -2.60 & 1.12 & 0.09 & - & - & - & - & - & - & - & - \\
\hline
\end{tabular}

${ }^{a}$ The nearest model grid point for site SBB fell onto land. Therefore, we used the nearest model grid point in the ocean to perform a consistent model-data-comparison. 
Table S4.

\begin{tabular}{|c|c|c|c|c|c|c|c|c|c|c|c|c|c|}
\hline \multirow[t]{2}{*}{ Province } & \multicolumn{3}{|c|}{ Plankton Tow Details } & \multicolumn{2}{|c|}{ N. pachyderma } & \multicolumn{2}{|c|}{ N. incompta } & \multicolumn{2}{|c|}{ G. bulloides } & \multicolumn{2}{|c|}{ G. ruber (white) } & \multicolumn{2}{|c|}{ T. sacculifer } \\
\hline & Site & $\begin{array}{r}\text { Latitude } \\
\left({ }^{\circ} \mathrm{N}\right)\end{array}$ & $\begin{array}{r}\text { Longitude } \\
\left({ }^{\circ} \mathrm{E}\right)\end{array}$ & $\begin{array}{c}\text { Tow }^{\mathrm{a}} \\
(\mathrm{m})\end{array}$ & $\underset{(\mathrm{m})}{\operatorname{PLAFOM} 2.0^{\mathrm{a}}}$ & $\begin{array}{c}\text { Tow }^{\mathrm{a}} \\
(\mathrm{m})\end{array}$ & $\begin{array}{c}\text { PLAFOM2.0 } \\
(\mathrm{m})\end{array}$ & $\begin{array}{c}\text { Tow }^{\mathrm{a}} \\
(\mathrm{m})\end{array}$ & $\underset{(\mathrm{m})}{\operatorname{PLAFOM} 2.0^{\mathrm{a}}}$ & $\begin{array}{c}\text { Tow }^{\mathrm{a}} \\
(\mathrm{m})\end{array}$ & $\underset{(\mathrm{m})}{\operatorname{PLAFOM} 2.0^{\mathrm{a}}}$ & $\begin{array}{c}\text { Tow }^{\mathrm{a}} \\
(\mathrm{m})\end{array}$ & $\underset{(\mathrm{m})}{\operatorname{PLAFOM} 2.0^{\mathrm{a}}}$ \\
\hline \multirow{11}{*}{$\frac{\pi}{0}$} & $93-36$ & 80.36 & -10.14 & $85 \pm 35$ & $55 \pm 35$ & - & - & - & - & - & - & - & - \\
\hline & PS78-25 & 78.83 & 7.00 & $85 \pm 55$ & $50 \pm 35$ & - & - & - & - & - & - & - & - \\
\hline & PS78-44 & 78.83 & 0.08 & $80 \pm 40$ & $45 \pm 35$ & - & - & - & - & - & - & - & - \\
\hline & PS78-75 & 78.83 & -3.92 & $70 \pm 40$ & $45 \pm 35$ & - & - & - & - & - & - & - & - \\
\hline & PS55-025 & 75.00 & -10.58 & $90 \pm 70$ & $45 \pm 35$ & - & - & - & - & - & - & - & - \\
\hline & PS55-043 & 75.00 & 0.36 & $60 \pm 40$ & $45 \pm 30$ & - & - & - & - & - & - & - & - \\
\hline & PS55-063 & 75.00 & 10.65 & $85 \pm 65$ & $45 \pm 30$ & - & - & $55 \pm 25$ & $25 \pm 15$ & - & - & - & - \\
\hline & MN116 & 75.00 & -7.31 & $150 \pm 40$ & $45 \pm 30$ & - & - & - & - & - & - & - & - \\
\hline & MN2 & 70.00 & 3.40 & $170 \pm 215$ & $60 \pm 50$ & - & - & - & - & - & - & - & - \\
\hline & MN323 & 69.69 & 0.47 & $140 \pm 155$ & $55 \pm 45$ & - & - & - & - & - & - & - & - \\
\hline & MN314 & 67.54 & 5.58 & $125 \pm 60$ & $65 \pm 55$ & - & - & - & - & - & - & - & - \\
\hline \multirow{3}{*}{ 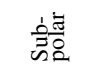 } & PAPA & 49.98 & -144.97 & $230 \pm 30$ & $80 \pm 35$ & $105 \pm 85$ & $60 \pm 35$ & $60 \pm 45$ & $70 \pm 40$ & - & - & - & - \\
\hline & 101 & 47.00 & -174.95 & $95 \pm 50$ & $60 \pm 40$ & $140 \pm 40$ & $55 \pm 35$ & $65 \pm 30$ & $50 \pm 35$ & - & - & - & - \\
\hline & 79 & 46.98 & 166.73 & $110 \pm 55$ & $55 \pm 40$ & $150 \pm 50$ & $25 \pm 15$ & $70 \pm 35$ & $30 \pm 25$ & - & - & - & - \\
\hline \multirow{5}{*}{ 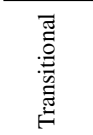 } & KNOT & 44.08 & 154.98 & $90 \pm 45$ & $70 \pm 45$ & $75 \pm 55$ & $20 \pm 10$ & $45 \pm 30$ & $30 \pm 25$ & - & - & - & - \\
\hline & $\# \mathrm{~B}^{\mathrm{b}}$ & 41.57 & 141.90 & $125 \pm 40$ & $80 \pm 40$ & $105 \pm 45$ & $70 \pm 45$ & $100 \pm 55$ & $75 \pm 45$ & $122 \pm 40$ & $5 \pm 5$ & - & - \\
\hline & & 41.15 & 143.38 & $85 \pm 40$ & $80 \pm 40$ & $35 \pm 25$ & $70 \pm 45$ & $40 \pm 30$ & $75 \pm 45$ & & & & 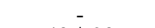 \\
\hline & MOC1-38 & 38.92 & -67.90 & - & - & - & - & $65 \pm 55$ & $75 \pm 40$ & $30 \pm 20$ & $35 \pm 20$ & $35 \pm 20$ & $40 \pm 20$ \\
\hline & $\#$ A & 36.02 & 141.78 & - & - & $25 \pm 20$ & $65 \pm 35$ & $25 \pm 25$ & $65 \pm 35$ & $20 \pm 20$ & $25 \pm 20$ & $25 \pm 20$ & $30 \pm 20$ \\
\hline \multirow{6}{*}{ 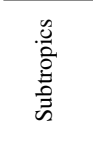 } & POS383-165 & 34.00 & -22.00 & - & - & $85 \pm 60$ & $80 \pm 45$ & $170 \pm 70$ & $75 \pm 40$ & $65 \pm 25$ & $30 \pm 25$ & $185 \pm 85$ & $50 \pm 30$ \\
\hline & MOC $1-28$ & 33.91 & -71.78 & - & - & - & - & - & - & $80 \pm 35$ & $50 \pm 30$ & $60 \pm 35$ & $50 \pm 30$ \\
\hline & POS383-175 & 33.15 & -22.00 & - & - & $85 \pm 55$ & $80 \pm 40$ & $95 \pm 50$ & $75 \pm 40$ & $65 \pm 25$ & $35 \pm 25$ & $190 \pm 65$ & $50 \pm 30$ \\
\hline & POS 247-1389 & 33.08 & -22.00 & - & - & $30 \pm 0$ & $80 \pm 45$ & $30 \pm 0$ & $80 \pm 45$ & $55 \pm 25$ & $50 \pm 30$ & $40 \pm 35$ & $55 \pm 30$ \\
\hline & MOC $1-23$ & 32.73 & -71.16 & - & - & - & - & $140 \pm 0$ & $70 \pm 35$ & $115 \pm 0$ & $45 \pm 25$ & $95 \pm 50$ & $50 \pm 30$ \\
\hline & $\# \mathrm{E}$ & 32.17 & 133.88 & - & - & $60 \pm 30$ & $80 \pm 40$ & - & - & $70 \pm 45$ & $30 \pm 20$ & $45 \pm 40$ & $35 \pm 25$ \\
\hline \multirow{14}{*}{ 菊 } & 920 & 16.09 & 52.70 & - & - & - & - & $65 \pm 65$ & $70 \pm 40$ & $40 \pm 45$ & $40 \pm 20$ & $20 \pm 20$ & $45 \pm 25$ \\
\hline & 310 & 16.02 & 52.73 & - & - & - & - & $30 \pm 35$ & $50 \pm 35$ & $180 \pm 100$ & $10 \pm 5$ & $180 \pm 85$ & $15 \pm 10$ \\
\hline & 313 & 15.91 & 53.02 & - & - & - & - & $70 \pm 80$ & $50 \pm 35$ & $30 \pm 35$ & $10 \pm 5$ & $30 \pm 30$ & $10 \pm 5$ \\
\hline & 917 & 15.89 & 52.97 & - & - & - & - & $90 \pm 65$ & $70 \pm 40$ & $75 \pm 50$ & $40 \pm 20$ & $20 \pm 15$ & $45 \pm 25$ \\
\hline & MOC63 & 2.92 & -140.20 & - & - & - & - & $15 \pm 10$ & $95 \pm 50$ & $20 \pm 15$ & $45 \pm 25$ & $25 \pm 15$ & $45 \pm 25$ \\
\hline & MOC65 & 2.05 & -141.49 & - & - & - & - & $25 \pm 15$ & $90 \pm 50$ & $35 \pm 25$ & $40 \pm 20$ & $25 \pm 15$ & $40 \pm 25$ \\
\hline & MOC 12 & 2.01 & -139.88 & - & - & - & - & $65 \pm 25$ & $80 \pm 45$ & $45 \pm 25$ & $45 \pm 25$ & $45 \pm 25$ & $40 \pm 25$ \\
\hline & MOC66 & 1.13 & -140.01 & - & - & - & - & $55 \pm 25$ & $85 \pm 45$ & $45 \pm 20$ & $35 \pm 20$ & $45 \pm 25$ & $35 \pm 20$ \\
\hline & MOC 15 & 0.00 & -140.07 & - & - & - & - & - & - & $20 \pm 10$ & $35 \pm 20$ & $25 \pm 15$ & $35 \pm 20$ \\
\hline & MOC69 & -1.05 & -139.97 & - & - & - & - & $25 \pm 15$ & $80 \pm 45$ & $25 \pm 15$ & $20 \pm 15$ & $25 \pm 15$ & $40 \pm 20$ \\
\hline & MOC20 & -2.02 & -140.16 & - & - & - & - & - & - & $35 \pm 15$ & $45 \pm 25$ & $40 \pm 20$ & $40 \pm 25$ \\
\hline & MOC71 & -2.33 & -140.32 & - & - & - & - & $45 \pm 25$ & $90 \pm 45$ & $35 \pm 25$ & $45 \pm 25$ & $35 \pm 25$ & $45 \pm 25$ \\
\hline & SO225-21-3 & -3.05 & -165.06 & - & - & - & - & $145 \pm 90$ & $55 \pm 30$ & $65 \pm 35$ & $55 \pm 30$ & $75 \pm 45$ & $55 \pm 30$ \\
\hline & MOC 72 & -3.21 & -140.25 & - & - & - & - & $40 \pm 20$ & $90 \pm 50$ & $35 \pm 15$ & $50 \pm 25$ & $35 \pm 20$ & $50 \pm 25$ \\
\hline \multirow{2}{*}{ 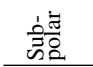 } & TNO57-16 & -50.12 & 5.75 & $70 \pm 10$ & $60 \pm 35$ & $70 \pm 10$ & $25 \pm 15$ & $80 \pm 5$ & $30 \pm 15$ & - & - & - & - \\
\hline & TNO57-13 & -53.18 & 5.13 & $85 \pm 60$ & $55 \pm 35$ & (1) & - & - & - & - & - & - & - \\
\hline \multirow{4}{*}{$\frac{\text { t }}{0}$} & AN98-O & -63.25 & 177.25 & $55 \pm 30$ & $60 \pm 35$ & - & - & - & - & - & - & - & - \\
\hline & AN99-O & -63.40 & 178.05 & $25 \pm 15$ & $60 \pm 40$ & - & - & - & - & - & - & - & - \\
\hline & AN01-O & -63.43 & 178.10 & $120 \pm 0$ & $60 \pm 40$ & - & - & - & - & - & - & - & - \\
\hline & ANOO-O & -63.53 & 178.38 & $95 \pm 50$ & $60 \pm 40$ & - & - & - & - & - & - & - & - \\
\hline
\end{tabular}

${ }^{\mathrm{a}} \mathrm{ALD} \pm \mathrm{VD}$ (in $\mathrm{m}$ ) of the planktonic foraminiferal species calculated after Rebotim et al. (2017) for the plankton tow samples and for PLAFOM2.0 (obtained at the nearest model grid points of the given plankton tow locations). Note that the values have been rounded to the nearest $5 \mathrm{~m}$.

$\mathrm{b}$ The nearest model grid point for site \#B fell onto the shelf. Therefore, we used the nearest model grid point in the open ocean to perform a consistent model-data-comparison. 


\section{References}

Asahi, H. and Takahashi, K.: A 9-year time-series of planktonic foraminifer fluxes and environmental change in the Bering sea and the central subarctic Pacific Ocean, 1990-1999, Progress in Oceanography, 72, 343-363, https://doi.org/10.1016/j.pocean.2006.03.021, 2007.

Bergami, C., Capotondi, L., Langone, L., Giglio, F., and Ravaioli, M.: Distribution of living planktonic foraminifera in the Ross Sea and the Pacific sector of the Southern Ocean (Antarctica), Marine Micropaleontology, 73, 37-48, https://doi.org/10.1016/j.marmicro.2009.06.007, 2009.

Curry, W. B., Ostermann, D. R., Guptha, M. V. S., and Ittekkot, V.: Foraminiferal production and monsoonal upwelling in the Arabian Sea: evidence from sediment traps, Geological Society, London, Special Publications, 64, 93-106, https://doi.org/10.1144/GSL.SP.1992.064.01.06, 1992.

10 Darling, K. F., Kucera, M., Wade, C. M., von Langen, P., and Pak, D.: Seasonal distribution of genetic types of planktonic foraminifer morphospecies in the Santa Barbara Channel and its paleoceanographic implications, Paleoceanography, 18, 1032, https://doi.org/10.1029/2001PA000723, 2003.

Deuser, W. G. and Ross, E. H.: Seasonally abundant planktonic foraminifera of the Sargasso Sea; succession, deep-water fluxes, isotopic compositions, and paleoceanographic implications, Journal of Foraminiferal Research, 19, 268-293, 1989.

Deuser, W. G., Ross, E. H., Hemleben, C., and Spindler, M.: Seasonal changes in species composition, numbers, mass, size, and isotopic composition of planktonic foraminifera settling into the deep Sargasso Sea, Palaeogeography, Palaeoclimatology, Palaeoecology, 33, 103127, https://doi.org/10.1016/0031-0182(81)90034-1, 1981.

Donner, B. and Wefer, G.: Flux and stable isotope composition of Neogloboquadrina pachyderma and other planktonic foraminifers in the Southern Ocean (Atlantic sector), Deep-Sea Research I, 41, 1733-1743, 1994.

Fairbanks, R. G., Wiebe, P. H., and Bé, A. W. H.: Vertical Distribution and Isotopic Composition of Living Planktonic Foraminifera in the Western North Atlantic, Science, 207, 61-63, 1980.

Fischer, G. and Wefer, G.: Long-term observation of particle fluxes in the eastern Atlantic: seasonality, changes of flux with depth and comparison with the sediment record, in: The South Atlantic: Present and Past Circulation, edited by Wefer, G., Berger, W. H., Siedler, G., and Webb, D., pp. 325-344, Springer, Berlin, Heidelberg, New York, 1996.

Iwasaki, S., Kimoto, K., Kuroyanagi, A., and Kawahata, H.: Horizontal and vertical distributions of planktic foraminifera in the subarctic Pacific, Marine Micropaleontology, 130, 1-14, https://doi.org/10.1016/j.marmicro.2016.12.001, 2017.

Jensen, S.: Planktische Foraminiferen im Europäischen Nordmeer: Verbreitung und Vertikalfluß sowie ihre Entwicklung während der letzten 15000 Jahre, Berichte Sonderforschungsbereich 313, Univ. Kiel, 75, 1-105, 1998.

Kincaid, E., Thunell, R. C., Le, J., Lange, C. B., Weinheimer, A. L., and Reid, F. M. H.: Planktonic foraminiferal fluxes in the Santa Barbara Basin: response to seasonal and interannual hydrographic changes, Deep-Sea Research Part II, 47, 1157-1176, https://doi.org/10.1016/S0967-0645(99)00140-X, 2000.

King, A. L. and Howard, W. R.: Seasonality of foraminiferal flux in sediment traps at Chatham rise, SW Pacific: Implications for paleotemperature estimates, Deep-Sea Research Part I, 48, 1687-1708, https://doi.org/10.1016/S0967-0637(00)00106-0, 2001.

Kohfeld, K. E., Fairbanks, R. G., Smith, S. L., and Walsh, I. D.: Neogloboquadrina pachyderma (sinistral coiling) as paleoceanographic tracers in polar oceans: Evidence from Northeast Water Polynya plankton tows, sediment traps, and surface sediments, Paleoceanography, 11, 679-699, 1996.

Kuroyanagi, A. and Kawahata, H.: Vertical distribution of living planktonic foraminifera in the seas around Japan, Marine Micropaleontology, 53, 173-196, https://doi.org/10.1016/j.marmicro.2004.06.001, 2004.

Kuroyanagi, A., Kawahata, H., Nishi, H., and Honda, M. C.: Seasonal changes in planktonic foraminifera in the northwestern North Pacific Ocean: sediment trap experiments from subarctic and subtropical gyres, Deep-Sea Research Part II, 49, 5627-5645, https://doi.org/10.1016/S0967-0645(02)00202-3, 2002. 
Locarnini, R. A., Mishonov, A. V., Antonov, J. I., Boyer, T. P., Garcia, H. E., Baranova, O. K., Zweng, M. M., Paver, C. R., Reagan, J. R., Johnson, D. R., Hamilton, M., and Seidov, D.: World Ocean Atlas 2013, Volume 1: Temperature, Silver Spring, MD, https://doi.org/10.1182/blood-2011-06-357442, 2013.

Mohiuddin, M. M., Nishimura, A., Tanaka, Y., and Shimamoto, A.: Regional and interannual productivity of biogenic components and planktonic foraminiferal fluxes in the northwestern Pacific Basin, Marine Micropaleontology, 45, 57-82, https://doi.org/10.1016/S03778398(01)00045-7, 2002.

Mohiuddin, M. M., Nishimura, A., Tanaka, Y., and Shimamoto, A.: Seasonality of biogenic particle and planktonic foraminifera fluxes: Response to hydrographic variability in the Kuroshio Extension, northwestern Pacific Ocean, Deep-Sea Research Part I, 51, 1659-1683, https://doi.org/10.1016/j.dsr.2004.06.002, 2004.

10 Mohiuddin, M. M., Nishimura, A., and Tanaka, Y.: Seasonal succession, vertical distribution, and dissolution of planktonic foraminifera along the Subarctic Front: Implications for paleoceanographic reconstruction in the northwestern Pacific, Marine Micropaleontology, 55, 129-156, https://doi.org/10.1016/j.marmicro.2005.02.007, 2005.

Mortyn, P. G. and Charles, C. D.: Planktonic foraminiferal depth habitat and $\delta^{18} \mathrm{O}$ calibrations: Plankton tow results from the Atlantic sector of the Southern Ocean, Paleoceanography, 18, 1037, https://doi.org/10.1029/2001PA000637, 2003.

Northcote, L. C. and Neil, H. L.: Seasonal variations in foraminiferal flux in the Southern Ocean, Campbell Plateau, New Zealand, Marine Micropaleontology, 56, 122-137, https://doi.org/10.1016/j.marmicro.2005.05.001, 2005.

Pados, T. and Spielhagen, R. F.: Species distribution and depth habitat of recent planktic foraminifera in Fram Strait, Arctic Ocean, Polar Research, 33, 22483, https://doi.org/10.3402/polar.v33.22483, 2014.

Peeters, F. J. C. and Brummer, G.-J. A.: The seasonal and vertical distribution of living planktic foraminifera in the NW Arabian Sea, Geological Society, London, Special Publications, 195, 463-497, https://doi.org/10.1144/GSL.SP.2002.195.01.26, 2002.

Rebotim, A., Voelker, A. H. L., Jonkers, L., Waniek, J. J., Meggers, H., Schiebel, R., Fraile, I., Schulz, M., and Kucera, M.: Factors controlling the depth habitat of planktonic foraminifera in the subtropical eastern North Atlantic, Biogeosciences, 14, 827-859, https://doi.org/10.5194/bg-14-827-2017, 2017.

Rippert, N., Nürnberg, D., Raddatz, J., Maier, E., Hathorne, E., Bijma, J., and Tiedemann, R.: Constraining foraminiferal calcification depths in the western Pacific warm pool, Marine Micropaleontology, 128, 14-27, https://doi.org/10.1016/j.marmicro.2016.08.004, 2016.

Sautter, L. R. and Thunell, R. C.: Seasonal succession of planktonic foraminifera, results from a four-year time-series sediment trap experiment in the Northeast Pacific, Journal of Foraminiferal Research, 19, 253-267, https://doi.org/10.2113/gsjfr.19.4.253, 1989.

Sautter, L. R. and Thunell, R. C.: Planktonic foraminiferal response to upwelling and seasonal hydrographic conditions: sediment trap results from San Pedro Basin, Southern California Bight, Journal of Foraminiferal Research, 21, 347-363, https://doi.org/10.2113/gsjfr.21.4.347, 1991.

Simstich, J., Sarnthein, M., and Erlenkeuser, H.: Paired $\delta^{18} \mathrm{O}$ signals of Neogloboquadrina pachyderma (s) and Turborotalita quinqueloba show thermal stratification structure in Nordic Seas, Marine Micropaleontology, 48, 107-125, https://doi.org/10.1016/S03778398(02)00165-2, 2003.

Stangeew, E.: Distribution and Isotopic Composition of Living Planktonic Foraminifera N. pachyderma (sinistral) and T. quinqueloba in the High Latitude North Atlantic, Ph.D. thesis, Christian-Albrechts-Universität zu Kiel, 2001.

Storz, D., Schulz, H., Waniek, J. J., Schulz-Bull, D. E., and Kučera, M.: Seasonal and interannual variability of the planktic foraminiferal flux in the vicinity of the Azores Current, Deep-Sea Research I, 56, 107-124, https://doi.org/10.1016/j.dsr.2008.08.009, 2009.

Watkins, J. M., Mix, A. C., and Wilson, J.: Living planktic foraminifera: tracers of circulation and productivity regimes in the central equatorial Pacific, Deep Sea Research II, 43, 1257-1282, 1996.

40 Watkins, J. M., Mix, A. C., and Wilson, J.: Living planktic foraminifera in the central tropical Pacific Ocean: Articulating the equatorial 'cold tongue' during La Niña, 1992, Marine Micropaleontology, 33, 157-174, https://doi.org/10.1016/S0377-8398(97)00036-4, 1998. 
Wolfteich, C. M.: Satellite-Derived Sea Surface Temperature, Mesoscale Variability, and Foraminiferal Production in the North Atlantic, M.Sc., Cambridge, MS, 1994. 